\title{
ENERGY CONSERVING DISCONTINUOUS GALERKIN SPECTRAL ELEMENT METHOD FOR THE VLASOV-POISSON SYSTEM
}

\author{
ÉRIC MADAULE**, MARCO RESTELLI ${ }^{\dagger}$, AND ERIC SONNENDRÜCKER ${ }^{\ddagger}$
}

\begin{abstract}
We propose a new, energy conserving, spectral element, discontinuous Galerkin method for the approximation of the Vlasov-Poisson system in arbitrary dimension, using Cartesian grids. The method is derived from the one proposed in [ACS12], with two modifications: energy conservation is obtained by a suitable projection operator acting on the solution of the Poisson problem, rather than by solving multiple Poisson problems, and all the integrals appearing in the finite element formulation are approximated with Gauss-Lobatto quadrature, thereby yielding a spectral element formulation. The resulting method has the following properties: exact energy conservation (up to errors introduced by the time discretization), stability (thanks to the use of upwind numerical fluxes), high order accuracy and high locality. For the time discretization, we consider both Runge-Kutta methods and exponential integrators, and show results for $1 \mathrm{D}$ and $2 \mathrm{D}$ cases (2D and 4D in phase space, respectively).
\end{abstract}

Key words. Vlasov-Poisson system, discontinuous Galerkin method, spectral element method

AMS subject classifications. 65N30, 65N35, 65M60, 65M70, 82D10

1. Introduction. The Vlasov-Poisson system describes the behaviour of a collisionless plasma subject to electrostatic effects in terms of the corresponding distribution function in phase space, thus representing one of the basic models in plasma physics. Due to the high dimensionality of the problem, efficient numerical techniques for the computation of approximate solutions are of paramount importance. Moreover, such techniques should ideally reproduce the main features of the system, namely: the absence of dissipation, the stability of the solution and the conservation of physical invariants such as the total number of particles, the total energy and the total momentum. In the literature, various approaches have been proposed, corresponding to different trade-offs concerning the fulfillment of these requirements. Semi-Lagrangian schemes emphasize computational efficiency, obtained using large time steps [SRBG99, FSB01, CMS10, RS11, GQ13, CGMQ14]. Conservative finite difference formulations, constructed in terms of the Hamiltonian formulation of the problem, result in conservation equations for the physical invariants of the discrete system which parallel those of the continuous one [Kra13]. Discontinuous Galerkin methods, finally, have been considered because of their locality, which allows optimal scaling in massively parallel computations, as well as their accuracy and flexibility in terms of the computational grid [ACS11, QS11, ACS12, HGMM12, CGM13, GQ13, AS12, CCZ14a, CCZ14b].

In this paper, we consider a discontinuous Galerkin (DG) spectral element method for the Vlasov-Poisson system with the introduction of a local reconstruction of the electric field which ensures exact discrete energy conservation up to errors introduced by the time discretization. This choice is motivated by the following considerations. The DG method provides the highest locality of the numerical discretization, repre-

\footnotetext{
*Institut Jean Lamour, Université de Lorraine, CNRS, UMR 7198, France (eric.madaule@univ-lorraine.fr).

† Max-Planck-Institut für Plasmaphysik, Boltzmannstr. 2, 85748 Garching, Germany (marco.restelli@ipp.mpg.de).

${ }^{\ddagger}$ Max-Planck-Institut für Plasmaphysik, Boltzmannstr. 2, 85748 Garching, Germany and TU Munich, Mathematics Center, Boltzmannstr. 3, 85747 Garching, Germany,
} (eric.sonnendruecker@ipp.mpg.de). 
senting an optimal choice for massively parallel computations, it combines the use of upwind numerical fluxes with high order accuracy, so that the numerical solution tends to be stable but not excessively dissipative, and it permits the use of nonuniform and even nonconforming grids [BSC11, MBGW13]. Moreover, the DG discretization ensures discrete conservation of the total number of particles in a very natural way. The spectral element version of the DG method is obtained by using a quadrature formula for the computation of the integrals appearing in the finite element formulation and by collocating the discrete degrees of freedom at the quadrature nodes [Boy01, Kop09] and, for high polynomial orders, results in a more efficient method compared to the standard DG one. The spectral element DG method has been used extensively for fluid dynamics computations where, as is the case for the Vlasov-Poisson system, the flow regime is strongly advection dominated but yet the solution does not develop shocks (for instance, see [RG09] for the case of atmospheric flows). The use of a special reconstruction of the electric field computed in the solution of the Poisson problem is introduced to compensate the spurious energy sources resulting from the upwind numerical flux employed in the DG method, as we discuss in details in the sequel of the paper; such a reconstruction is local (i.e. defined at the element level), in order to preserve the locality of the formulation, and consistent with the accuracy of the scheme, so that it does not degrade the overall convergence rate.

The resulting formulation can be regarded as a modification of the method proposed and analyzed in [ACS11, ACS12, AS12], the main modifications consisting in the facts that a) the method is of spectral element type and b) energy conservation is achieved using a local reconstruction of the electric field, instead of solving multiple Poisson problems. We notice that these two aspects are independent from each other, and in particular the energy conserving reconstruction of the electric field is also applicable in the framework of a standard DG method. We also observe that an alternative approach to obtain energy conserving formulations for the Vlasov-Poisson system can be found in [CCZ14a] (these ideas are then extended in [CCZ14b] to the Maxwell system), where the starting point for the numerical discretization is provided by Ampère's law and where energy conserving schemes are presented also for the completely discretized problem in space and time. The counterpart to this is that, by discretizing Ampère's law, charge conservation is not preserved for the numerical solution and most of the time marching schemes discussed in [CCZ14a] are implicit; in this respect, the pros and the cons of the method of [CCZ14a] are dual compared with those of the scheme discussed in the present paper.

After discretizing the Vlasov-Poisson system in space with the spectral element DG formulation, we obtain an Ordinary Differential Equation (ODE) which is then integrated in time following the classical method of lines. Typically, this is done with explicit Runge-Kutta (RK) methods, along the lines of the RKDG method introduced in the nowadays classical series of papers [CS91, CS89, CLS89, CHS90, CS98b]. This leads to an accurate and stable formulation, which computational cost however tends to be adversely affected by a somewhat stringent stability condition on the time-step, especially for high order polynomial spaces. A possible alternative to this strategy is represented by the use of of exponential time integrators [Law67, SSS09], and we present some preliminary comparisons between RK and exponential time marching schemes in the present paper. An alternative approach, which we postpone for further investigation, is the use of semi-Lagrangian DG methods [RBS06, QS11].

The remaining of the paper is organized as follows. In section 2, the VlasovPoisson system is introduced. The DG numerical discretization is discussed in 3, which 
mostly follows the ideas of [ACS11, ACS12]. Section 4 is devoted to the illustration of the local reconstruction strategy for the electric field. Section 5 then addresses all the aspects related with the spectral element formulation. In section 6 we summarize the time marching schemes considered for the time integration of the resulting ODE system. The resulting numerical scheme is validated numerically in section 7 , and finally some conclusions are drawn in section 8.

2. The Vlasov-Poisson system. In $d$ spatial dimensions, the Vlasov-Poisson system is

$$
\begin{aligned}
\partial_{t} f+\boldsymbol{v} \cdot \nabla_{\boldsymbol{x}} f-\boldsymbol{E} \cdot \nabla_{\boldsymbol{v}} f & =0 \\
\nabla_{\boldsymbol{x}} \cdot \boldsymbol{E} & =1-\rho,
\end{aligned}
$$

where $f=f(t, \boldsymbol{x}, \boldsymbol{v})$ is the particle distribution function, $\boldsymbol{E}=\nabla_{\boldsymbol{x}} \Phi$ is the electric field, $\Phi=\Phi(t, \boldsymbol{x})$ being the electrostatic potential, and $\rho=\rho(t, \boldsymbol{x})$ is the particle density. Equation (2.1), supplemented with an initial condition $f(0, \boldsymbol{x}, \boldsymbol{v})=f_{0}(\boldsymbol{x}, \boldsymbol{v})$, must be solved for times $t \in(0, T]$ and phase space coordinates $(\boldsymbol{x}, \boldsymbol{v}) \in \Omega_{\boldsymbol{x}} \times \Omega_{\boldsymbol{v}}=\Omega$. Here we assume that $\Omega_{\boldsymbol{x}}=\left(0, L_{\boldsymbol{x}}\right)^{d}$ and $\Omega_{\boldsymbol{v}}=\left(-\frac{L_{v}}{2}, \frac{L_{v}}{2}\right)^{d}$ and enforce periodic boundary conditions on both $\partial \Omega_{\boldsymbol{x}}$ and $\partial \Omega_{\boldsymbol{v}}$; vector components in $\Omega_{\boldsymbol{x}}$ and $\Omega_{\boldsymbol{v}}$ are denoted by a Greek superscript, such as $x^{\alpha}$ or $v^{\beta}$, for $1 \leq \alpha, \beta \leq d$. From a physical viewpoint, the periodic boundary condition represents a natural assumption for $\Omega_{\boldsymbol{x}}$, while the proper choice for the velocity space should be considering $\Omega_{\boldsymbol{v}}=\mathbb{R}^{d}$. However, for numerical computations, the velocity space must be truncated, which is acceptable provided that $L_{\boldsymbol{v}}$ is sufficiently large, and in this case the choice of the boundary condition on $\partial \Omega_{\boldsymbol{v}}$ is not relevant. Assuming periodic conditions then simplifies both the presentation and the numerical assessment of the conservation properties of the method. Concerning the particle density $\rho$, it is related to the distribution function by

$$
\rho(t, \boldsymbol{x})=\int_{\Omega_{\boldsymbol{v}}} f(t, \boldsymbol{x}, \boldsymbol{v}) \mathrm{d} \boldsymbol{v}
$$

In terms of the electric potential, $(2.1)_{2}$ can be written as

$$
\Delta_{x} \Phi=1-\rho
$$

which, together with the periodic boundary conditions on $\partial \Omega_{\boldsymbol{x}}$, requires the compatibility condition

$$
\int_{\Omega_{\boldsymbol{x}}} \rho(t, \boldsymbol{x}) \mathrm{d} \boldsymbol{x}=\iint_{\Omega} f(t, \boldsymbol{x}, \boldsymbol{v}) \mathrm{d} \boldsymbol{x} \mathrm{d} \boldsymbol{v}=L_{\boldsymbol{x}}^{d} .
$$

Since, as it will be discussed shortly, (2.1) implies that the integrals appearing in (2.4) are constant in time, it suffices to assume that (2.4) is satisfied for the initial condition $f_{0}$. Moreover, to provide a unique definition of the electrostatic potential, we require

$$
\int_{\Omega_{\boldsymbol{x}}} \Phi(t, \boldsymbol{x}) \mathrm{d} \boldsymbol{x}=0,
$$

thereby completing the definition of the continuous problem.

The Vlasov-Poisson system is characterized by a number of conservation properties, which we summarize here since one of the focuses of this article is investigating to 
what extent they are preserved by the numerical discretization. Let us first consider the weak form of the Vlasov equation, obtained multiplying $(2.1)_{1}$ by a test function $u=u(\boldsymbol{x}, \boldsymbol{v})$ and integrating over the phase space,

$$
\frac{d}{d t} \iint_{\Omega} f u \mathrm{~d} \boldsymbol{x} \mathrm{d} \boldsymbol{v}-\iint_{\Omega} \boldsymbol{v} f \cdot \nabla_{\boldsymbol{x}} u \mathrm{~d} \boldsymbol{x} \mathrm{d} \boldsymbol{v}+\iint_{\Omega} \boldsymbol{E} f \cdot \nabla_{\boldsymbol{v}} u \mathrm{~d} \boldsymbol{x} \mathrm{d} \boldsymbol{v}=0 .
$$

Taking now $u=1$ we obtain

$$
\iint_{\Omega} f \mathrm{~d} \boldsymbol{x} \mathrm{d} \boldsymbol{v}=\text { const }
$$

representing the physical constraint that no particles are created or destroyed. As anticipated, this also shows that the integrals appearing in (2.4) are constant in time.

Taking $u=v^{\alpha}$, for $\alpha=1, \ldots, d$, and using $(2.1)_{2}$, we arrive at

$$
\iint_{\Omega} \boldsymbol{v} f \mathrm{~d} \boldsymbol{x} \mathrm{d} \boldsymbol{v}=\text { const }
$$

representing the fact that the total momentum of the system, which is isolated from external forces, is conserved.

Taking $u=\frac{v^{2}}{2}+\Phi$, and using again $(2.1)_{2}$, we arrive at

$$
\iint_{\Omega} \frac{v^{2}}{2} f \mathrm{~d} \boldsymbol{x} \mathrm{d} \boldsymbol{v}+\frac{1}{2} \int_{\Omega_{\boldsymbol{x}}} E^{2} \mathrm{~d} \boldsymbol{x}=\text { const }
$$

representing the fact that the total energy of the system is conserved.

Two additional properties follow from the fact that, by virtue of $(2.1)_{1}$, the particle distribution function is constant along the trajectories of the vector field $(\boldsymbol{v}, \boldsymbol{E})$ in the phase space. It follows that

$$
\iint_{\Omega}|f|^{p} \mathrm{~d} \boldsymbol{x} \mathrm{d} \boldsymbol{v}=\text { const }, \quad 1 \leq p \leq \infty
$$

and

$$
\min _{\Omega} f_{0} \leq f(t, \boldsymbol{x}, \boldsymbol{v}) \leq \max _{\Omega} f_{0} \quad \forall t>0, \quad \forall(\boldsymbol{x}, \boldsymbol{v}) \in \Omega .
$$

Equation (2.10) corresponds to the conservation of the system entropy, while (2.11) guarantees that the distribution function is nonnegative, provided that such is the initial condition.

Various results concerning the well-posedness of the Vlasov-Poisson system, as well as the regularity of the solution, are summarized in [ACS11, ACS12].

3. Discontinuous Galerkin discretization of the Vlasov-Poisson system. Relevant references concerning the introduction and the analysis of the DG method are in [RH73, JP86, CS91, CS89, CLS89, CHS90, CS98b] for hyperbolic problems and [CS98a, CCPS01, ABCM02, CGL09] for elliptic problems. Concerning the specific application to the Vlasov-Poisson system, the DG method has then been studied in [ACS11, ACS12, HGMM12, CGM13, AS12]; in particular, error estimates of order $k+1$, where $k$ is the polynomial degree of the local finite element spaces, are shown in [ACS11, ACS12] for the particle distribution function and the electric field in the $L^{2}$ norm. The presentation of the method given in this section closely follows [ACS12]. 
3.1. Notation. Let us first introduce two tessellations $\mathcal{T}_{\boldsymbol{x}}$ and $\mathcal{T}_{\boldsymbol{v}}$ of $\Omega_{\boldsymbol{x}}$ and $\Omega_{\boldsymbol{v}}$, respectively, composed of rectangular elements $K_{\boldsymbol{x}}$ and $K_{\boldsymbol{v}}$; the element boundaries will be denoted by $\partial K_{\boldsymbol{x}}$ and $\partial K_{\boldsymbol{v}}$. The tessellation $\mathcal{T}$ for the phase space domain $\Omega$ is then composed of all the elements $K=K_{\boldsymbol{x}} \times K_{\boldsymbol{v}}$ resulting from the Cartesian product of two arbitrary elements $K_{\boldsymbol{x}} \in \mathcal{T}_{\boldsymbol{x}}$ and $K_{\boldsymbol{v}} \in \mathcal{T}_{\boldsymbol{v}}$, with boundary

$$
\partial K=\partial_{\boldsymbol{x}} K \cup \partial_{\boldsymbol{v}} K=\left(\partial K_{\boldsymbol{x}} \times K_{\boldsymbol{v}}\right) \cup\left(K_{\boldsymbol{x}} \times \partial K_{\boldsymbol{v}}\right) .
$$

The outward pointing normal vector on the boundary of any element is denoted by $\boldsymbol{n}$. The edges of the tessellations are indicated with $e_{\boldsymbol{x}}, e_{\boldsymbol{v}}$ and $e$ for $\mathcal{T}_{\boldsymbol{x}}, \mathcal{T}_{\boldsymbol{v}}$ and $\mathcal{T}$, respectively, and their collections are $\mathcal{E}_{\boldsymbol{x}}, \mathcal{E}_{\boldsymbol{v}}$ and $\mathcal{E}$. For edges $e \in \mathcal{E}$ we also distinguish between $e_{\boldsymbol{v}, \boldsymbol{x}}=e_{\boldsymbol{x}} \times K_{\boldsymbol{v}}$ and $e_{\boldsymbol{x}, \boldsymbol{v}}=K_{\boldsymbol{x}} \times e_{\boldsymbol{v}}$. We assume that the tessellations are conforming and, although this might not be strictly required, that the edges are parallel to the coordinate axes, so that, for instance, $x^{\alpha}=$ const on $e_{\boldsymbol{x}} \in \mathcal{E}_{\boldsymbol{x}}$ for some $1 \leq \alpha \leq d$. In view of definition (4.7), we also assume that the hyperplane $\boldsymbol{v}=0$ is entirely covered by sides $e_{\boldsymbol{x}, \boldsymbol{v}} \in \mathcal{E}$, i.e. no element $K$ lies "across" this hyperplane, so that the sign of each velocity component does not change within $K$. It is also convenient to introduce a notation for a stripe in phase space, local in space and global in velocity; we thus let

$$
S_{K_{x}}=K_{\boldsymbol{x}} \times \Omega_{\boldsymbol{v}}, \quad \partial S_{K_{\boldsymbol{x}}}=\partial K_{\boldsymbol{x}} \times \Omega_{\boldsymbol{v}}
$$

The standard notation for averages and jumps is then introduced as follows (see for instance $[\mathrm{ABCM} 02])$ : considering a generic function $\chi_{h}$, piecewise continuous on $\mathcal{T}_{\boldsymbol{x}}$, for a given element $K_{\boldsymbol{x}}$, edge $e_{\boldsymbol{x}} \subset \partial K_{\boldsymbol{x}}$ and point $\boldsymbol{x} \in e_{\boldsymbol{x}}$, we define

$$
\chi_{h}\left(\boldsymbol{x}^{i n t\left(K_{\boldsymbol{x}}\right)}\right)=\lim _{\substack{\boldsymbol{y} \rightarrow \boldsymbol{x} \\ \boldsymbol{y} \in K_{\boldsymbol{x}}}} \chi_{h}(\boldsymbol{y}) .
$$

This definition can be extended to a vector valued function $\boldsymbol{r}_{h}$ by applying it componentwise. Then, for $e_{\boldsymbol{x}}=\partial K_{\boldsymbol{x}} \cap \partial K_{\boldsymbol{x}}^{\prime}, \boldsymbol{x} \in e_{\boldsymbol{x}}$, let

$$
\begin{aligned}
\left\{\chi_{h}\right\}(\boldsymbol{x}) & =\frac{1}{2} \chi_{h}\left(\boldsymbol{x}^{i n t\left(K_{\boldsymbol{x}}\right)}\right)+\frac{1}{2} \chi_{h}\left(\boldsymbol{x}^{i n t\left(K_{\boldsymbol{x}}^{\prime}\right)}\right) \\
\llbracket \chi_{h} \rrbracket(\boldsymbol{x}) & =\boldsymbol{n} \chi_{h}\left(\boldsymbol{x}^{i n t\left(K_{\boldsymbol{x}}\right)}\right)+\boldsymbol{n}^{\prime} \chi_{h}\left(\boldsymbol{x}^{i n t\left(K_{\boldsymbol{x}}^{\prime}\right)}\right) \\
\left\{\boldsymbol{r}_{h}\right\}(\boldsymbol{x}) & =\frac{1}{2} \boldsymbol{r}_{h}\left(\boldsymbol{x}^{\text {int }\left(K_{\boldsymbol{x}}\right)}\right)+\frac{1}{2} \boldsymbol{r}_{h}\left(\boldsymbol{x}^{i n t\left(K_{\boldsymbol{x}}^{\prime}\right)}\right) \\
\llbracket \boldsymbol{r}_{h} \rrbracket(\boldsymbol{x}) & =\boldsymbol{n} \cdot \boldsymbol{r}_{h}\left(\boldsymbol{x}^{\operatorname{int}\left(K_{\boldsymbol{x}}\right)}\right)+\boldsymbol{n}^{\prime} \cdot \boldsymbol{r}_{h}\left(\boldsymbol{x}^{\operatorname{int}\left(K_{\boldsymbol{x}}^{\prime}\right)}\right),
\end{aligned}
$$

where $\boldsymbol{n}$ and $\boldsymbol{n}^{\prime}$ are the outward pointing normals at $\boldsymbol{x}$ on $\partial K_{\boldsymbol{x}}$ and $\partial K_{\boldsymbol{x}}^{\prime}$, respectively, with $\boldsymbol{n}^{\prime}=-\boldsymbol{n}$. Considering the product of a scalar and a vector valued function, the following identities are easily verified:

$$
\left\{\chi_{h} \boldsymbol{r}_{h}\right\} \cdot \boldsymbol{n}=\left\{\chi_{h}\right\}\left\{\boldsymbol{r}_{h}\right\} \cdot \boldsymbol{n}+\frac{1}{4} \llbracket \boldsymbol{r}_{h} \rrbracket \llbracket \chi_{h} \rrbracket \cdot \boldsymbol{n}
$$

and

$$
\llbracket \chi_{h} \boldsymbol{r}_{h} \rrbracket=\left\{\chi_{h}\right\} \llbracket \boldsymbol{r}_{h} \rrbracket+\llbracket \chi_{h} \rrbracket \cdot\left\{\boldsymbol{r}_{h}\right\},
$$

which are often used in the analysis of DG formulations. These definitions can be extended to elements in $\mathcal{T}_{\boldsymbol{v}}$ and $\mathcal{T}$. 
Having defined the tessellation of the computational domain, the next element required to define the spectral element DG formulation is a basis for the local polynomial space where the discrete solution is sought, as well as some quadrature formulae. We denote by $\left\{\hat{\xi}_{i}^{1 \mathrm{D}, k}\right\}_{i=0}^{k}$ the Legendre-Gauss-Lobatto (LGL) points on the interval $[-1,1]$, defined as the roots of the polynomial $\left(1-\xi^{2}\right) \hat{\psi}_{k}^{\prime}(\xi)$, where $\hat{\psi}_{k}$ is the $k$-th Legendre polynomial on $[-1,1]$ (see [CHQZ06, CHQZ07]), and let $\left\{\hat{\varphi}_{i}^{1 \mathrm{D}, k}\right\}_{i=0}^{k}$ be the Lagrangian basis of the polynomial space $\mathbb{P}_{k}([-1,1])$ associated with such points. Next, we introduce the $(k+1)^{d}$ multivariate polynomials

$$
\hat{\varphi}_{i}(\boldsymbol{\xi})=\prod_{\alpha=1}^{d} \hat{\varphi}_{i^{\alpha}}^{1 \mathrm{D}, k}\left(\xi^{\alpha}\right)
$$

where $\boldsymbol{\xi}=\left(\xi^{1}, \ldots, \xi^{d}\right)$, with $\xi^{\alpha} \in[-1,1]$, and $\boldsymbol{i}$ is the multi-index $i^{1}, \ldots, i^{d}$, with $0 \leq i^{\alpha} \leq k$, as well as the $(k+2)(k+1)^{d-1}$ multivariate polynomials

$$
\hat{\varphi}_{i_{+}^{\alpha}}(\boldsymbol{\xi})=\hat{\varphi}_{i^{\alpha}}^{1 \mathrm{D}, k+1}\left(\xi^{\alpha}\right) \prod_{\substack{\beta=1 \\ \beta \neq \alpha}}^{d} \hat{\varphi}_{i^{\beta}}^{1 \mathrm{D}, k}\left(\xi^{\beta}\right),
$$

where $\boldsymbol{i}_{+}^{\alpha}$ is the multi-index $i^{1}, \ldots, i^{d}$, with $0 \leq i^{\beta} \leq k$ if $\beta \neq \alpha$ and $0 \leq i^{\alpha} \leq k+1$. The polynomials $\hat{\varphi}_{\boldsymbol{i}}$ and $\hat{\varphi}_{\boldsymbol{i}^{\alpha}}$ clearly represent the Lagrangian bases associated with the nodes $\boldsymbol{\xi}_{\boldsymbol{i}}=\left(\hat{\xi}_{i^{1}}^{1 \mathrm{D}, k}, \ldots, \hat{\xi}_{i^{d}}^{1 \mathrm{D}, k}\right)$ and $\boldsymbol{\xi}_{\boldsymbol{i}_{+}^{\alpha}}=\left(\hat{\xi}_{i^{1}}^{1 \mathrm{D}, k}, \ldots, \hat{\xi}_{i^{\alpha}}^{1 \mathrm{D}, k+1}, \ldots, \hat{\xi}_{i^{d}}^{1 \mathrm{D}, k}\right)$, respectively. Now, for a generic element $K_{\boldsymbol{x}}$, let $\mathcal{F}_{K_{\boldsymbol{x}}}$ be a transformation mapping $[-1,1]^{d}$ onto $\bar{K}_{\boldsymbol{x}}$, define

$$
\varphi_{K_{x}, i}(\boldsymbol{x})= \begin{cases}\left(\hat{\varphi}_{\boldsymbol{i}} \circ \mathcal{F}_{K_{x}}^{-1}\right)(\boldsymbol{x}), & \boldsymbol{x} \in \bar{K}_{\boldsymbol{x}} \\ 0, & \boldsymbol{x} \notin \bar{K}_{\boldsymbol{x}}\end{cases}
$$

and denote by $\mathbb{Q}_{k}\left(K_{\boldsymbol{x}}\right)$ the space generated by the functions $\varphi_{K_{\boldsymbol{x}}, \boldsymbol{i}}(\boldsymbol{x})$ for all the possible values of the multi-index. Similarly, define

$$
\varphi_{K_{\boldsymbol{x}}, \boldsymbol{i}_{+}^{\alpha}}(\boldsymbol{x})= \begin{cases}\left(\hat{\varphi}_{\boldsymbol{i}_{+}^{\alpha}} \circ \mathcal{F}_{K_{\boldsymbol{x}}}^{-1}\right)(\boldsymbol{x}), & \boldsymbol{x} \in \bar{K}_{\boldsymbol{x}} \\ 0, & \boldsymbol{x} \notin \bar{K}_{\boldsymbol{x}}\end{cases}
$$

and denote by $\mathbb{Q}_{k_{+}^{\alpha}}\left(K_{\boldsymbol{x}}\right)$ the space generated by these functions for all the possible values of the multi-index. The following finite element spaces can now be introduced:

$$
\begin{gathered}
V_{h}^{k}\left(\mathcal{T}_{\boldsymbol{x}}\right)=\left\{\chi_{h} \in L^{2}\left(\Omega_{\boldsymbol{x}}\right):\left.\chi_{h}\right|_{K_{\boldsymbol{x}}} \in \mathbb{Q}_{k}\left(K_{\boldsymbol{x}}\right)\right\} \\
\boldsymbol{W}_{h}^{k}\left(\mathcal{T}_{\boldsymbol{x}}\right)=\left\{\boldsymbol{r}_{h} \in\left(L^{2}\left(\Omega_{\boldsymbol{x}}\right)\right)^{d}:\left.r_{h}^{\alpha}\right|_{K_{\boldsymbol{x}}} \in \mathbb{Q}_{k}\left(K_{\boldsymbol{x}}\right), 1 \leq \alpha \leq d\right\},
\end{gathered}
$$

and

$$
\boldsymbol{R} \boldsymbol{T}_{h}^{k}\left(\mathcal{T}_{\boldsymbol{x}}\right)=\left\{\boldsymbol{r}_{h} \in\left(L^{2}\left(\Omega_{\boldsymbol{x}}\right)\right)^{d}:\left.r_{h}^{\alpha}\right|_{K_{\boldsymbol{x}}} \in \mathbb{Q}_{k_{+}^{\alpha}}\left(K_{\boldsymbol{x}}\right), 1 \leq \alpha \leq d\right\},
$$

where the first two spaces are the standard piecewise polynomial spaces, while the third one is the Raviart-Thomas space introduced in [RT77]. The same construction 
of course can be repeated for $\mathcal{T}_{\boldsymbol{v}}$ and $\mathcal{T}$. In this paper, we always assume that $k \geq 2$; this is not a real limitation, since spectral element formulations typically use highorder polynomials anyway.

The LGL points define a quadrature rule on $[-1,1]$ with weights

$$
\hat{w}_{i}^{1 \mathrm{D}, k}=\frac{2}{k(k+1) \hat{\psi}_{k}\left(\hat{\xi}_{i}^{1 \mathrm{D}, k}\right)} .
$$

A tensor product, multidimensional quadrature rule can then be obtained introducing the weights

$$
\hat{w}_{i}=\prod_{\alpha=1}^{d} \hat{w}_{i^{\alpha}}^{1 \mathrm{D}, k}
$$

and approximating integrals as

$$
\int_{K_{\boldsymbol{x}}} \chi_{h}(\boldsymbol{x}) \mathrm{d} \boldsymbol{x} \approx \int_{K_{\boldsymbol{x}}, w} \chi_{h}(\boldsymbol{x}) \mathrm{d} \boldsymbol{x}=\frac{\left|K_{\boldsymbol{x}}\right|}{2^{d}} \sum_{\boldsymbol{i}} \hat{w}_{\boldsymbol{i}} \chi_{\boldsymbol{i}},
$$

where the summation is extended over all the possible values of the multi-index, $\left|K_{x}\right|$ is the volume of the element and $\chi_{\boldsymbol{i}}=\chi_{h}\left(\mathcal{F}_{K_{\boldsymbol{x}}}\left(\boldsymbol{\xi}_{\boldsymbol{i}}\right)\right)$. Notice that, when $\chi_{h} \in V_{h}^{k}\left(\mathcal{T}_{\boldsymbol{x}}\right)$, the values $\chi_{i}$ are the coefficients with respect to the Lagrangian basis $\varphi_{K_{x}, i}$. A similar construction allows to approximate integrals on $e_{\boldsymbol{x}} \subset \partial K_{\boldsymbol{x}}$. Clearly, this quadrature formula can be applied also for the integration of components of vector valued functions belonging to $\boldsymbol{W}_{h}^{k}\left(\mathcal{T}_{\boldsymbol{x}}\right)$, while for functions in $\boldsymbol{R} \boldsymbol{T}_{h}^{k}\left(\mathcal{T}_{\boldsymbol{x}}\right)$ it is convenient to introduce the quadrature formula

$$
\int_{K_{x}} r_{h}^{\alpha}(\boldsymbol{x}) \mathrm{d} \boldsymbol{x} \approx \int_{K_{\boldsymbol{x}}, w_{+}^{\alpha}} r_{h}^{\alpha}(\boldsymbol{x}) \mathrm{d} \boldsymbol{x}=\frac{\left|K_{\boldsymbol{x}}\right|}{2^{d}} \sum_{\boldsymbol{i}_{+}^{\alpha}} \hat{w}_{\boldsymbol{i}_{+}^{\alpha}} r_{\boldsymbol{i}_{+}^{\alpha}}^{\alpha},
$$

with

$$
\hat{w}_{i_{+}^{\alpha}}=\hat{w}_{i^{\alpha}}^{1 \mathrm{D}, k+1} \prod_{\substack{\beta=1 \\ \beta \neq \alpha}}^{d} \hat{w}_{i^{\beta}}^{1 \mathrm{D}, k}
$$

and $r_{\boldsymbol{i}_{+}^{\alpha}}^{\alpha}=r_{h}^{\alpha}\left(\mathcal{F}_{K_{\boldsymbol{x}}}\left(\boldsymbol{\xi}_{\boldsymbol{i}_{+}^{\alpha}}\right)\right)$. Notice that in both cases $\boldsymbol{r}_{h} \in \boldsymbol{W}_{h}^{k}\left(\mathcal{T}_{\boldsymbol{x}}\right)$ and $\boldsymbol{r}_{h} \in \boldsymbol{R} \boldsymbol{T}_{h}^{k}\left(\mathcal{T}_{\boldsymbol{x}}\right)$ the normal flux $\left.\boldsymbol{n} \cdot \boldsymbol{r}_{h}\right|_{e_{\boldsymbol{x}}}$ is a polynomial of degree $k$.

3.2. DG discretization of the Vlasov equation. The DG discretization of the Vlasov equation is obtained multiplying $(2.1)_{1}$ by a test function $u_{h}(\boldsymbol{x}, \boldsymbol{v})$, integrating over $K \in \mathcal{T}$, replacing the exact solution with the corresponding numeric approximation and integrating by parts. Taking $u_{h} \in V_{h}^{k}(\mathcal{T})$ and $f_{h}(t, \cdot, \cdot) \in V_{h}^{k}(\mathcal{T})$ $\forall t \in[0, T]$ we obtain

$$
\begin{aligned}
\iint_{K} \partial_{t} f_{h} u_{h} \mathrm{~d} \boldsymbol{x} \mathrm{d} \boldsymbol{v} & -\iint_{K} \boldsymbol{v} f_{h} \cdot \nabla_{\boldsymbol{x}} u_{h} \mathrm{~d} \boldsymbol{x} \mathrm{d} \boldsymbol{v}+\int_{\partial_{\boldsymbol{x}} K} \boldsymbol{n} \cdot \widehat{\boldsymbol{v f}} u_{h} \mathrm{~d} \boldsymbol{\sigma} \mathrm{d} \boldsymbol{v} \\
& +\iint_{K} \tilde{\boldsymbol{E}}_{h} f_{h} \cdot \nabla_{\boldsymbol{v}} u_{h} \mathrm{~d} \boldsymbol{x} \mathrm{d} \boldsymbol{v}-\int_{\partial_{\boldsymbol{v}} K} \boldsymbol{n} \cdot \widehat{\boldsymbol{E f}} u_{h} \mathrm{~d} \boldsymbol{x} \mathrm{d} \boldsymbol{\tau}=0,
\end{aligned}
$$


where the hat symbol denotes the so-called numerical fluxes, to be defined shortly, and $\tilde{\boldsymbol{E}}_{h}$ is an approximation of the electric field which precise form will be discussed in $\S 4$ and which will play an important role in the construction of an energy conserving discretization. According to (3.1), $\boldsymbol{\sigma}$ and $\boldsymbol{\tau}$ denote the integration variables on $\partial K_{\boldsymbol{x}}$ and $\partial K_{\boldsymbol{v}}$, respectively, where $K=K_{\boldsymbol{x}} \times K_{\boldsymbol{v}}$.

Concerning the numerical fluxes, we consider here two alternatives: centered fluxes and upwind fluxes. In the space coordinate, these are defined by

$$
\widehat{\boldsymbol{v f f}}^{\text {centered }}=\left\{\boldsymbol{v} f_{h}\right\}, \quad \widehat{\boldsymbol{v f f}}^{\text {upwind }}=\left\{\boldsymbol{v} f_{h}\right\}+\frac{1}{2}|\boldsymbol{v} \cdot \boldsymbol{n}| \llbracket f_{h} \rrbracket,
$$

while in the velocity coordinate these are

$$
\widehat{\boldsymbol{E f}}^{\text {centered }}=\left\{\boldsymbol{E} f_{h}\right\}, \quad \widehat{\boldsymbol{E f f}}^{\text {upwind }}=\left\{\boldsymbol{E} f_{h}\right\}-\frac{1}{2}|\boldsymbol{E} \cdot \boldsymbol{n}| \llbracket f_{h} \rrbracket .
$$

Whatever definition is used, it is important to realize that these numerical fluxes are single valued quantities on $\partial_{\boldsymbol{x}} K$ and $\partial_{\boldsymbol{v}} K$, respectively. Notice that centered and upwind fluxes can be regarded as special cases of a weighted upwind flux

$$
\widehat{\boldsymbol{v f f}}^{\omega}=\left\{\boldsymbol{v} f_{h}\right\}+\frac{\omega}{2}|\boldsymbol{v} \cdot \boldsymbol{n}| \llbracket f_{h} \rrbracket, \quad \widehat{\boldsymbol{E f}}^{\omega}=\left\{\boldsymbol{E} f_{h}\right\}-\frac{\omega}{2}|\boldsymbol{E} \cdot \boldsymbol{n}| \llbracket f_{h} \rrbracket
$$

for an upwinding parameter $\omega \in[0,1]$.

Notice that, taking $u_{h}=\left.1\right|_{K}$, the characteristic function of the element $K$, we obtain the local mass balance

$$
\frac{d}{d t} \iint_{K} f_{h} \mathrm{~d} \boldsymbol{x} \mathrm{d} \boldsymbol{v}=-\int_{\partial_{\boldsymbol{x}} K} \boldsymbol{n} \cdot \widehat{\boldsymbol{v f}} \mathrm{d} \boldsymbol{\sigma} \mathrm{d} \boldsymbol{v}+\int_{\partial_{\boldsymbol{v}} K} \boldsymbol{n} \cdot \widehat{\boldsymbol{E} f} \mathrm{~d} \boldsymbol{x} \mathrm{d} \boldsymbol{\tau},
$$

and, since the numerical fluxes are single valued on $\partial K$, the change in the number of particles in $K$ is exactly balanced by the changes in the neighbouring elements, so that the total number of particles is constant. In other terms, the DG method is by its nature a flux form method.

3.3. DG discretization of the Poisson equation. To obtain the DG discretization of the Poisson equation, we first rewrite $(2.1)_{2}$ in mixed form

$$
\begin{aligned}
\nabla_{\boldsymbol{x}} \cdot \boldsymbol{E} & =1-\rho \\
\boldsymbol{E}-\nabla_{\boldsymbol{x}} \Phi & =0 .
\end{aligned}
$$

Then, multiplying (3.9) $)_{1}$ and $(3.9)_{2}$ by $\boldsymbol{z}_{h}(\boldsymbol{x})$ and $p_{h}(\boldsymbol{x})$, respectively, integrating over $K_{\boldsymbol{x}} \in \mathcal{T}_{\boldsymbol{x}}$, replacing the exact solution with the corresponding numeric approximation and integrating by parts we obtain

$$
\begin{array}{r}
\int_{K_{\boldsymbol{x}}} \boldsymbol{E}_{h} \cdot \boldsymbol{z}_{h} \mathrm{~d} \boldsymbol{x}-\int_{K_{\boldsymbol{x}}} \nabla_{\boldsymbol{x}} \Phi_{h} \cdot \boldsymbol{z}_{h} \mathrm{~d} \boldsymbol{x}-\int_{\partial K_{\boldsymbol{x}}}\left(\widehat{\Phi}-\Phi_{h}\right) \boldsymbol{n} \cdot \boldsymbol{z}_{h} \mathrm{~d} \boldsymbol{\sigma}=0 \\
\int_{K_{\boldsymbol{x}}} \boldsymbol{E}_{h} \cdot \nabla_{\boldsymbol{x}} p_{h} \mathrm{~d} \boldsymbol{x}-\int_{\partial K_{\boldsymbol{x}}} \boldsymbol{n} \cdot \widehat{\boldsymbol{E}} p_{h} \mathrm{~d} \boldsymbol{\sigma}=-\int_{K_{\boldsymbol{x}}}\left(1-\rho_{h}\right) p_{h} \mathrm{~d} \boldsymbol{x}
\end{array}
$$

with

$$
\rho_{h}(t, \boldsymbol{x})=\int_{\Omega_{v}} f_{h}(t, \boldsymbol{x}, \boldsymbol{v}) \mathrm{d} \boldsymbol{v} .
$$


The choice of the finite element spaces for the primal variable $\Phi_{h}$ and the dual variable $\boldsymbol{E}_{h}$ plays an important role in the definition of the characteristics of the resulting formulation. We consider here $\Phi_{h}, p_{h} \in V_{h}^{k}\left(\mathcal{T}_{\boldsymbol{x}}\right) \cap L_{0}^{2}\left(\Omega_{\boldsymbol{x}}\right)$, where $L_{0}^{2}\left(\Omega_{\boldsymbol{x}}\right)$ is the space of square integrable functions on $\Omega_{\boldsymbol{x}}$ with vanishing integral, while for the dual variable we consider two possibilities: $\boldsymbol{E}_{h}, \boldsymbol{z}_{h} \in \boldsymbol{W}_{h}^{k}\left(\mathcal{T}_{\boldsymbol{x}}\right)$ and $\boldsymbol{E}_{h}, \boldsymbol{z}_{h} \in \boldsymbol{R} \boldsymbol{T}_{h}^{k}\left(\mathcal{T}_{\boldsymbol{x}}\right)$.

The numerical fluxes are defined as

$$
\widehat{\boldsymbol{E}}=\left\{\boldsymbol{E}_{h}\right\}-c_{11} \llbracket \Phi_{h} \rrbracket, \quad \widehat{\Phi}=\left\{\Phi_{h}\right\},
$$

where $c_{11} \geq 0$. Notice that, compared with the general Local Discontinuous Galerkin (LDG) method analyzed in [CCPS01] and considered in [ACS11, ACS12, AS12] we restrict ourself to the case $\boldsymbol{c}_{12}=0, c_{22}=0$.

Notice also that, in (3.10), the differential operator $\nabla_{\boldsymbol{x}}$ acts on the unknown $\Phi_{h}$ in the first equation and on the test function $p_{h}$ in the second equation, which corresponds to what is sometimes referred to as "strong form" and "weak form" respectively, see for instance [GR08]. The two forms are of course equivalent, but differences can arise substituting the exact integration appearing in (3.10) with numerical integration. The chosen form is motivated by the fact that it will ensure energy conservation even in the case of approximate integration.

The choice of the finite element space for the dual variable, together with the condition $c_{11} \geq 0$, deserves some comments. The case $\boldsymbol{E}_{h} \in \boldsymbol{W}_{h}^{k}\left(\mathcal{T}_{\boldsymbol{x}}\right)$ is the standard LDG method analyzed in [CCPS01, ABCM02], and requires $c_{11}>0$. The case $\boldsymbol{E}_{h} \in$ $\boldsymbol{R} \boldsymbol{T}_{h}^{k}\left(\mathcal{T}_{\boldsymbol{x}}\right)$ is introduced here because this space will allow us to define an energy conserving formulation in $\S 4$. In this second case, we assume $c_{11} \geq 0$, which requires a minor modification of the stability proof of the scheme, namely Proposition 2.1 of [CCPS01]. Thus, we state the following

LEMма 3.1. Let $\boldsymbol{E}_{h}, \boldsymbol{z}_{h} \in \boldsymbol{R} \boldsymbol{T}_{h}^{k}\left(\mathcal{T}_{\boldsymbol{x}}\right)$ and $\Phi_{h}, p_{h} \in V_{h}^{k}\left(\mathcal{T}_{\boldsymbol{x}}\right) \cap L_{0}^{2}\left(\Omega_{\boldsymbol{x}}\right)$ and let $c_{11} \geq 0$. Then (3.10) admits a unique solution.

Proof. Substituting the numerical fluxes (3.12) in (3.10), summing for all $K_{\boldsymbol{x}} \in \mathcal{T}_{\boldsymbol{x}}$ and changing sign in $(3.10)_{2}$ we have

$$
\begin{gathered}
\int_{\Omega_{\boldsymbol{x}}} \boldsymbol{E}_{h} \cdot \boldsymbol{z}_{h} \mathrm{~d} \boldsymbol{x}-\int_{\Omega_{\boldsymbol{x}}} \nabla_{\boldsymbol{x}} \Phi_{h} \cdot \boldsymbol{z}_{h} \mathrm{~d} \boldsymbol{x}+\sum_{e_{\boldsymbol{x}} \in \mathcal{E}_{\boldsymbol{x}}} \int_{e_{\boldsymbol{x}}} \llbracket \Phi_{h} \rrbracket \cdot\left\{\boldsymbol{z}_{h}\right\} \mathrm{d} \boldsymbol{\sigma}=0 \\
-\int_{\Omega_{\boldsymbol{x}}} \boldsymbol{E}_{h} \cdot \nabla_{\boldsymbol{x}} p_{h} \mathrm{~d} \boldsymbol{x}+\sum_{e_{\boldsymbol{x}} \in \mathcal{E}_{\boldsymbol{x}}} \int_{e_{\boldsymbol{x}}}\left(\left\{\boldsymbol{E}_{h}\right\}-c_{11} \llbracket \Phi_{h} \rrbracket\right) \cdot \llbracket p_{h} \rrbracket \mathrm{d} \boldsymbol{\sigma}=\int_{\Omega_{\boldsymbol{x}}}\left(1-\rho_{h}\right) p_{h} \mathrm{~d} \boldsymbol{x} .
\end{gathered}
$$

Due to the linearity and finite dimensionality of the problem, we need to show that $\rho_{h}=1$ implies $\boldsymbol{E}_{h}=0, \Phi_{h}=0$. Indeed, taking $\boldsymbol{z}_{h}=\boldsymbol{E}_{h}$ and $p_{h}=\Phi_{h}$ and subtracting the resulting equations yields

$$
\int_{\Omega_{\boldsymbol{x}}} E_{h}^{2} \mathrm{~d} \boldsymbol{x}+\sum_{e_{\boldsymbol{x}} \in \mathcal{E}_{\boldsymbol{x}}} \int_{e_{\boldsymbol{x}}} c_{11} \llbracket \Phi_{h} \rrbracket^{2} \mathrm{~d} \boldsymbol{\sigma}=0,
$$

from which we can deduce $\boldsymbol{E}_{h}=0$ and

$$
-\int_{\Omega_{\boldsymbol{x}}} \nabla_{\boldsymbol{x}} \Phi_{h} \cdot \boldsymbol{z}_{h} \mathrm{~d} \boldsymbol{x}+\sum_{e_{\boldsymbol{x}} \in \mathcal{E}_{\boldsymbol{x}}} \int_{e_{\boldsymbol{x}}} \llbracket \Phi_{h} \rrbracket \cdot\left\{\boldsymbol{z}_{h}\right\} \mathrm{d} \boldsymbol{\sigma}=0 .
$$

As discussed in Lemma 6 of [RT77], we can choose $\boldsymbol{z}_{h} \in \boldsymbol{R} \boldsymbol{T}_{h}^{k}\left(\mathcal{T}_{\boldsymbol{x}}\right) \cap H\left(\operatorname{div}, \Omega_{\boldsymbol{x}}\right)$ specifying the following degrees of freedom: 
- the values of $\boldsymbol{z}_{h} \cdot \boldsymbol{n}=\left\{\boldsymbol{z}_{h}\right\} \cdot \boldsymbol{n}$ at $k+1$ distinct points on each $e_{\boldsymbol{x}}$

- the moments

$$
\int_{K_{x}} z_{h}^{\alpha} \pi^{\alpha} \mathrm{d} \boldsymbol{x}
$$

on each element $K_{x}$, with

$$
\pi^{\alpha}(\boldsymbol{x})=\prod_{\beta=1}^{d}\left(x^{\beta}\right)^{k_{\alpha, \beta},} \quad\left\{\begin{array}{cc}
0 \leq k_{\alpha, \beta} \leq k-1, & \alpha=\beta \\
0 \leq k_{\alpha, \beta} \leq k, & \alpha \neq \beta .
\end{array}\right.
$$

This implies that, for any $\Phi_{h} \in V_{h}^{k}\left(\mathcal{T}_{\boldsymbol{x}}\right)$, we can choose $\boldsymbol{z}_{h}$ so that

$$
\int_{\Omega_{\boldsymbol{x}}} \nabla_{\boldsymbol{x}} \Phi_{h} \cdot \boldsymbol{z}_{h} \mathrm{~d} \boldsymbol{x}=\int_{\Omega_{\boldsymbol{x}}}\left(\nabla_{\boldsymbol{x}} \Phi_{h}\right)^{2} \mathrm{~d} \boldsymbol{x}, \quad \int_{e_{\boldsymbol{x}}} \llbracket \Phi_{h} \rrbracket \cdot\left\{\boldsymbol{z}_{h}\right\} \mathrm{d} \boldsymbol{\sigma}=\int_{e_{\boldsymbol{x}}} \llbracket \Phi_{h} \rrbracket^{2} \mathrm{~d} \boldsymbol{\sigma},
$$

which shows $\Phi_{h}=$ const. Since $\Phi_{h} \in L_{0}^{2}\left(\Omega_{\boldsymbol{x}}\right)$, we conclude $\Phi_{h}=0$.

Concerning the accuracy of the method obtained with $\boldsymbol{E}_{h} \in \boldsymbol{R} \boldsymbol{T}_{h}^{k}\left(\mathcal{T}_{\boldsymbol{x}}\right)$, we observe that, for $c_{11}>0$, the error estimates provided in [CCPS01], Theorem 2.2, namely convergence of order $k$ and $k+1$ for $\left\|\boldsymbol{E}-\boldsymbol{E}_{h}\right\|_{L^{2}}$ and $\left\|\Phi-\Phi_{h}\right\|_{L^{2}}$, respectively, remain valid, since they are based on the inclusion property (2.14) of such reference, while for the case $c_{11}=0$ we provide in $\S 7.1$ some numerical results showing comparable accuracy with the $c_{11}>0$ case.

An interesting observation can be made comparing the method given by (3.10) with $\boldsymbol{E}_{h} \in \boldsymbol{R} \boldsymbol{T}_{h}^{k}\left(\mathcal{T}_{\boldsymbol{x}}\right), c_{11}=0$, and the classical Raviart-Thomas formulation [RT77], especially considering the hybridizable formulation analyzed in [AB85]. In fact, the two methods employ the same finite element spaces, but different definitions of the numerical fluxes. In the hybridizable Raviart-Thomas method, the numerical flux for the primary variable is treated as an additional unknown, setting $\hat{\Phi}=\lambda$, which is then determined by the condition that $\boldsymbol{E}_{h} \cdot \boldsymbol{n}$ shall be single valued on each edge (i.e. $\boldsymbol{E}_{h} \in H\left(\operatorname{div}, \Omega_{\boldsymbol{x}}\right)$ ), while in our case $\hat{\Phi}$ is a combination of the two local values $\left.\Phi_{h}\right|_{\partial K_{x}}$ and $\left.\Phi_{h}\right|_{\partial K_{x}^{\prime}}$, in the spirit of the DG method, and in general we have $\boldsymbol{E}_{h} \notin H\left(\operatorname{div}, \Omega_{\boldsymbol{x}}\right)$. We refer to [CGL09] for a detailed analysis of the relations between DG schemes and hybridizable schemes.

4. An energy conserving formulation. In this section, we first analyze to which extent the energy balance (2.9) is reproduced by the discrete method, and then we show how it is possible to obtain an exactly energy conserving spatial discretization by taking advantage of the freedom in the choice of the finite element spaces for $\boldsymbol{E}_{h}$ and $\Phi_{h}$ and in the definition of $\tilde{\boldsymbol{E}}_{h}$.

The total energy appearing in (2.9) is the sum of two contributions: the kinetic energy and the electrostatic energy, and the conversion between these two energies takes place because of the work done by the electrostatic force. To obtain a local in space, kinetic energy balance for the discrete problem, let us take $u_{h}=v^{2} / 2$ in (3.4) (this is always possible since we assume that $u_{h} \in V_{h}^{k}(\mathcal{T})$ with $k \geq 2$ ) and add the resulting equations for $K \in S_{K_{x}}$, for a generic element $K_{x}$. Since the chosen test function is independent from $\boldsymbol{x}$, we have $\nabla_{\boldsymbol{x}} u_{h}=0$, and since it is continuous, the integrals over $\partial_{\boldsymbol{v}} K$ cancel. The resulting equation is

$$
\frac{d}{d t} \iint_{S_{K_{\boldsymbol{x}}}} f_{h} \frac{v^{2}}{2} \mathrm{~d} \boldsymbol{x} \mathrm{d} \boldsymbol{v}=-\int_{\partial S_{K_{\boldsymbol{x}}}} \boldsymbol{n} \cdot \widehat{\boldsymbol{v} f} \frac{v^{2}}{2} \mathrm{~d} \boldsymbol{\sigma} \mathrm{d} \boldsymbol{v}-\iint_{S_{K_{\boldsymbol{x}}}} f_{h} \tilde{\boldsymbol{E}}_{h} \cdot \boldsymbol{v} \mathrm{d} \boldsymbol{x} \mathrm{d} \boldsymbol{v},
$$


representing the fact that the kinetic energy contained in $K_{\boldsymbol{x}}$ varies because of the fluxes of kinetic energy on $\partial K_{\boldsymbol{x}}$ and because of the work done by the electrostatic force. To obtain the corresponding electrostatic energy balance, one needs to consider $u_{h}=\Phi_{h}$ in (3.4), which is indeed possible given our choice of finite element spaces. Then using the fact that $\Phi_{h}$ does not depend on $\boldsymbol{v}$, and thus it is also continuous on $\partial_{v} K$, we obtain

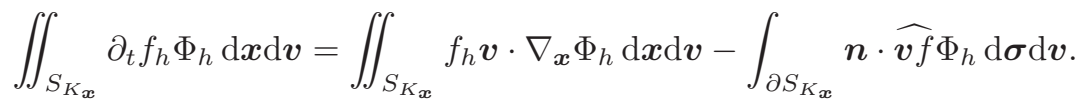

The first term can be expressed in terms of the time derivative of the electrostatic energy considering the Poisson equation. In particular, taking the time derivative of $(3.10)_{2}$, setting $p_{h}=\Phi_{h}$ and using (3.11), we have

$$
\begin{aligned}
\iint_{S_{K_{\boldsymbol{x}}}} \partial_{t} f_{h} \Phi_{h} \mathrm{~d} \boldsymbol{x} \mathrm{d} \boldsymbol{v} & =\int_{K_{\boldsymbol{x}}} \partial_{t} \rho_{h} \Phi_{h} \mathrm{~d} \boldsymbol{x} \\
& =\int_{K_{\boldsymbol{x}}} \partial_{t} \boldsymbol{E}_{h} \cdot \nabla_{\boldsymbol{x}} \Phi_{h} \mathrm{~d} \boldsymbol{x}-\int_{\partial K_{\boldsymbol{x}}} \boldsymbol{n} \cdot \partial_{t} \widehat{\boldsymbol{E}} \Phi_{h} \mathrm{~d} \boldsymbol{\sigma} .
\end{aligned}
$$

The first term in the right-hand side can be computed taking $\boldsymbol{z}_{h}=\partial_{t} \boldsymbol{E}_{h}$ in $(3.10)_{1}$, so that

$$
\begin{aligned}
\iint_{S_{K_{\boldsymbol{x}}}} \partial_{t} f_{h} \Phi_{h} \mathrm{~d} \boldsymbol{x} \mathrm{d} \boldsymbol{v}= & \frac{d}{d t} \int_{K_{\boldsymbol{x}}} \frac{E_{h}^{2}}{2} \mathrm{~d} \boldsymbol{x}-\int_{\partial K_{\boldsymbol{x}}} \boldsymbol{n} \cdot \partial_{t} \widehat{\boldsymbol{E}} \Phi_{h} \mathrm{~d} \boldsymbol{\sigma} \\
& -\int_{\partial K_{\boldsymbol{x}}} \boldsymbol{n} \cdot \partial_{t} \boldsymbol{E}\left(\widehat{\Phi}-\Phi_{h}\right) \mathrm{d} \boldsymbol{\sigma}
\end{aligned}
$$

Substituting in (4.2) yields the local electrostatic energy balance

$$
\begin{aligned}
\frac{d}{d t} \int_{K_{\boldsymbol{x}}} \frac{E_{h}^{2}}{2} \mathrm{~d} \boldsymbol{x} & =\iint_{S_{K_{\boldsymbol{x}}}} f_{h} \boldsymbol{v} \cdot \nabla_{\boldsymbol{x}} \Phi_{h} \mathrm{~d} \boldsymbol{x} \mathrm{d} \boldsymbol{v} \\
& +\int_{\partial K_{\boldsymbol{x}}} \boldsymbol{n} \cdot\left(\partial_{t} \boldsymbol{E}_{h} \widehat{\Phi}-\partial_{t} \boldsymbol{E}_{h} \Phi_{h}+\partial_{t} \widehat{\boldsymbol{E}} \Phi_{h}-\int_{\Omega_{\boldsymbol{v}}} \widehat{\boldsymbol{v} f} \Phi_{h} \mathrm{~d} \boldsymbol{v}\right) \mathrm{d} \boldsymbol{\sigma} .
\end{aligned}
$$

The local energy balance can thus be obtained adding (4.1) and (4.3), so that

$$
\begin{array}{r}
\frac{d}{d t}\left[\iint_{S_{K_{\boldsymbol{x}}}} f_{h} \frac{v^{2}}{2} \mathrm{~d} \boldsymbol{x} \mathrm{d} \boldsymbol{v}+\int_{K_{\boldsymbol{x}}} \frac{E_{h}^{2}}{2} \mathrm{~d} \boldsymbol{x}\right]=\iint_{S_{K_{\boldsymbol{x}}}} f_{h} \boldsymbol{v} \cdot\left(\nabla_{\boldsymbol{x}} \Phi_{h}-\tilde{\boldsymbol{E}}_{h}\right) \mathrm{d} \boldsymbol{x} \mathrm{d} \boldsymbol{v} \\
-\int_{\partial S_{K_{\boldsymbol{x}}}} \boldsymbol{n} \cdot \widehat{\boldsymbol{v} f} \frac{v^{2}}{2} \mathrm{~d} \boldsymbol{\sigma} \mathrm{d} \boldsymbol{v} \\
+\int_{\partial K_{\boldsymbol{x}}} \boldsymbol{n} \cdot\left(\partial_{t} \boldsymbol{E}_{h} \widehat{\Phi}-\partial_{t} \boldsymbol{E}_{h} \Phi_{h}+\partial_{t} \widehat{\boldsymbol{E}} \Phi_{h}-\int_{\Omega_{\boldsymbol{v}}} \widehat{\boldsymbol{v} f} \Phi_{h} \mathrm{~d} \boldsymbol{v}\right) \mathrm{d} \boldsymbol{\sigma} .
\end{array}
$$

Equation (4.4) shows that, in general, spurious energy sources and sinks can be introduced in the discrete energy balance for two reasons: the fact that $\tilde{\boldsymbol{E}}_{h} \neq \nabla_{\boldsymbol{x}} \Phi_{h}$ in the first term of the right-hand side, so that the contributions associated with the work of the electrostatic force do not balance exactly, and the fact that the boundary fluxes of electrostatic energy, i.e. the third term in the right-hand side, are not single valued on $\partial K_{\boldsymbol{x}}$. Notice that the boundary fluxes of kinetic energy, i.e. the second term in the right-hand side of (4.4), are by construction a single valued quantity on $\partial K_{\boldsymbol{x}}$, so no spurious terms in the energy balance appear because of these fluxes. We now consider various methods ensuring an exact energy balance. 
4.1. Centered discretization of the Vlasov equation. The simplest case is the case of a centered flux in the Vlasov equation.

THEOREM 4.1. Consider the discrete problem (3.4), (3.10) and (3.11) with $\boldsymbol{E}_{h} \in$ $\boldsymbol{W}_{h}^{k}\left(\mathcal{T}_{\boldsymbol{x}}\right)$ or $\boldsymbol{E}_{h} \in \boldsymbol{R} \boldsymbol{T}_{h}^{k}\left(\mathcal{T}_{\boldsymbol{x}}\right)$, assume that

$$
\widehat{\boldsymbol{v f f}}=\left\{\boldsymbol{v} f_{h}\right\} \quad \text { on } \quad \partial_{\boldsymbol{x}} K
$$

and let

$$
\tilde{\boldsymbol{E}}_{h}=\boldsymbol{E}_{h}
$$

Then the following total energy is conserved:

$$
\mathscr{E}=\iint_{\Omega} f_{h} \frac{v^{2}}{2} d \boldsymbol{x} d \boldsymbol{v}+\int_{\Omega_{\boldsymbol{x}}} \frac{E_{h}^{2}}{2} d \boldsymbol{x}+\sum_{e_{\boldsymbol{x}} \in \mathcal{E}_{\boldsymbol{x}}} \frac{1}{2} \int_{e_{\boldsymbol{x}}} c_{11} \llbracket \Phi_{h} \rrbracket^{2} d \boldsymbol{\sigma} .
$$

Proof. Consider the energy balance (4.4). For a fixed value $\boldsymbol{v}$, the function $\boldsymbol{v} f_{h}(\boldsymbol{x}, \boldsymbol{v})$ belongs to $\boldsymbol{W}_{h}^{k}\left(\mathcal{T}_{\boldsymbol{x}}\right)$ (and thus also to $\boldsymbol{R} \boldsymbol{T}_{h}^{k}\left(\mathcal{T}_{\boldsymbol{x}}\right)$ ), so that we can take $\boldsymbol{z}_{h}=$ $\boldsymbol{v} f_{h}$ in $(3.10)_{1}$ and obtain, using (4.5),

$$
\begin{aligned}
\iint_{S_{K_{\boldsymbol{x}}}} f_{h} \boldsymbol{v} \cdot\left(\nabla_{\boldsymbol{x}} \Phi_{h}-\tilde{\boldsymbol{E}}_{h}\right) \mathrm{d} \boldsymbol{x} \mathrm{d} \boldsymbol{v} & =\int_{\Omega_{\boldsymbol{v}}}\left(\int_{K_{\boldsymbol{x}}} \boldsymbol{v} f_{h} \cdot\left(\nabla_{\boldsymbol{x}} \Phi_{h}-\boldsymbol{E}_{h}\right) \mathrm{d} \boldsymbol{x}\right) \mathrm{d} \boldsymbol{v} \\
& =-\int_{\Omega_{\boldsymbol{v}}}\left(\int_{\partial K_{\boldsymbol{x}}} \boldsymbol{v} f_{h} \cdot \boldsymbol{n}\left(\widehat{\Phi}-\Phi_{h}\right) \mathrm{d} \boldsymbol{\sigma}\right) \mathrm{d} \boldsymbol{v} \\
& =\int_{\partial K_{\boldsymbol{x}}} \boldsymbol{n} \cdot\left(\int_{\Omega_{\boldsymbol{v}}} \boldsymbol{v} f_{h}\left(\Phi_{h}-\widehat{\Phi}\right) \mathrm{d} \boldsymbol{v}\right) \mathrm{d} \boldsymbol{\sigma}
\end{aligned}
$$

This suggests the definition of

$$
\boldsymbol{q}^{\mathscr{E}}=\left(\widehat{\Phi}-\Phi_{h}\right)\left(\partial_{t} \boldsymbol{E}_{h}-\int_{\Omega_{\boldsymbol{v}}} \boldsymbol{v} f_{h} \mathrm{~d} \boldsymbol{v}\right)+\Phi_{h}\left(\partial_{t} \widehat{\boldsymbol{E}}-\int_{\Omega_{\boldsymbol{v}}} \widehat{\boldsymbol{v f f}} \mathrm{d} \boldsymbol{v}\right)-\int_{\Omega_{\boldsymbol{v}}} \widehat{\boldsymbol{v} f} \frac{v^{2}}{2} \mathrm{~d} \boldsymbol{v}
$$

so that (4.4) can be rewritten as

$$
\frac{d}{d t}\left[\iint_{S_{K_{\boldsymbol{x}}}} f_{h} \frac{v^{2}}{2} \mathrm{~d} \boldsymbol{x} \mathrm{d} \boldsymbol{v}+\int_{K_{\boldsymbol{x}}} \frac{E_{h}^{2}}{2} \mathrm{~d} \boldsymbol{x}\right]=\int_{\partial K_{\boldsymbol{x}}} \boldsymbol{n} \cdot \boldsymbol{q}^{\mathscr{E}} \mathrm{d} \boldsymbol{\sigma}
$$

and summing over all the elements $K_{\boldsymbol{x}} \in \mathcal{T}_{\boldsymbol{x}}$

$$
\frac{d}{d t}\left[\iint_{\Omega} f_{h} \frac{v^{2}}{2} \mathrm{~d} \boldsymbol{x} \mathrm{d} \boldsymbol{v}+\int_{\Omega_{\boldsymbol{x}}} \frac{E_{h}^{2}}{2} \mathrm{~d} \boldsymbol{x}\right]=\sum_{e_{\boldsymbol{x}} \in \mathcal{E}_{\boldsymbol{x}}} \int_{e_{\boldsymbol{x}}} \llbracket \boldsymbol{q}^{\mathscr{E}} \rrbracket \mathrm{d} \boldsymbol{\sigma}
$$

The thesis follows observing that the jump of the energy flux vanishes, up to a term which can be included in the definition of the total energy. In fact, using (3.3) and the expressions of the numerical fluxes, we have

$$
\begin{aligned}
\llbracket \boldsymbol{q}^{\mathscr{E}} \rrbracket & =\left(\widehat{\Phi}-\left\{\Phi_{h}\right\}\right)\left(\llbracket \partial_{t} \boldsymbol{E}_{h} \rrbracket-\int_{\Omega_{\boldsymbol{v}}} \llbracket \boldsymbol{v} f_{h} \rrbracket \mathrm{d} \boldsymbol{v}\right) \\
& +\llbracket \Phi_{h} \rrbracket \cdot\left(\partial_{t} \widehat{\boldsymbol{E}}-\left\{\partial_{t} \boldsymbol{E}_{h}\right\}-\int_{\Omega_{\boldsymbol{v}}}\left(\widehat{\boldsymbol{v} f}-\left\{\boldsymbol{v} f_{h}\right\}\right) \mathrm{d} \boldsymbol{v}\right)=-c_{11} \frac{\partial}{\partial t} \frac{\llbracket \Phi_{h} \rrbracket^{2}}{2}
\end{aligned}
$$

$\square$ 
4.2. Upwind discretization of the Vlasov equation. The definition of $\tilde{\boldsymbol{E}}_{h}$ is less trivial in the case of an upwind numerical flux in the Vlasov equation. In this case, it is useful to introduce, for a generic element $K=K_{\boldsymbol{x}} \times K_{\boldsymbol{v}}$, the tensor

$$
\mathcal{H}_{K}=\left[\begin{array}{lll}
\eta_{K}^{1} & & \\
& \ddots & \\
& & \eta_{K}^{d}
\end{array}\right]
$$

where

$$
\eta_{K}^{\alpha}=\eta_{K}^{\alpha}(\boldsymbol{x}, \boldsymbol{v})= \begin{cases}\hat{\eta}^{+}\left(\hat{\xi}^{\alpha}(\boldsymbol{x})\right), & v^{\alpha} \geq 0 \\ \hat{\eta}^{-}\left(\hat{\xi}^{\alpha}(\boldsymbol{x})\right), & v^{\alpha}<0\end{cases}
$$

with $\hat{\boldsymbol{\xi}}(\boldsymbol{x})=\mathcal{F}_{K_{\boldsymbol{x}}}^{-1}(\boldsymbol{x})$, and, for $\xi \in[-1,1]$,

$$
\hat{\eta}^{+}(\xi)=1+\xi, \quad \hat{\eta}^{-}(\xi)=1-\xi
$$

For two elements $K=K_{\boldsymbol{x}} \times K_{\boldsymbol{v}}, K^{\prime}=K_{\boldsymbol{x}}^{\prime} \times K_{\boldsymbol{v}}$, with $e_{\boldsymbol{x}}=\partial K_{\boldsymbol{x}} \cap \partial K_{\boldsymbol{x}}^{\prime}$ and $e=e_{\boldsymbol{x}} \times K_{\boldsymbol{v}}=\partial K \cap \partial K^{\prime}$, definition (4.7) ensures that a) for fixed $\boldsymbol{v}, \mathcal{H}_{K} \boldsymbol{v}$ is a vector in $\boldsymbol{R} \boldsymbol{T}_{h}^{0}\left(\mathcal{T}_{\boldsymbol{x}}\right)$, i.e. the $\alpha$-th component is an affine function of $x^{\alpha}$ and the remaining ones are constant; b) since $\boldsymbol{v}$ is a continuous function on $\mathcal{T}$,

$$
\left.\left(\left\{\mathcal{H}_{K} \boldsymbol{v}\right\}\right)^{\alpha}\right|_{e}=\left.\left\{\eta_{K}^{\alpha}\right\} v^{\alpha}\right|_{e}=\left.\left.v^{\alpha}\right|_{e} \quad \Longrightarrow \quad\left\{\mathcal{H}_{K} \boldsymbol{v}\right\}\right|_{e}=\left.\boldsymbol{v}\right|_{e}
$$

and c) assuming that on $e_{\boldsymbol{x}}$ the normal vector is $\boldsymbol{e}_{\alpha}$, the unit vector directed along the $\alpha$-th coordinate axis, one has

$$
\left.\llbracket \mathcal{H}_{K} \boldsymbol{v} \rrbracket\right|_{e}=\left.2\left(\eta_{K}^{\alpha}-\eta_{K^{\prime}}^{\alpha}\right) v^{\alpha}\right|_{e}=\left.2|\boldsymbol{v} \cdot \boldsymbol{n}|\right|_{e} .
$$

With these definitions, we can now prove the following theorem, representing the counterpart of Theorem 4.1 in the upwing case.

Theorem 4.2. Consider the discrete problem (3.4), (3.10) and (3.11) with $\boldsymbol{E}_{h} \in$ $\boldsymbol{R} \boldsymbol{T}_{h}^{k}\left(\mathcal{T}_{\boldsymbol{x}}\right)$, assume

$$
\widehat{\boldsymbol{v f}}=\left\{\boldsymbol{v} f_{h}\right\}+\frac{1}{2}|\boldsymbol{v} \cdot \boldsymbol{n}| \llbracket f_{h} \rrbracket \quad \text { on } \quad \partial_{\boldsymbol{x}} K
$$

and let

$$
\left.\tilde{\boldsymbol{E}}_{h}\right|_{K}=\left.\mathcal{H}_{K} \boldsymbol{E}_{h}\right|_{K}+\left.\left(\mathcal{I}-\mathcal{H}_{K}\right) \nabla_{\boldsymbol{x}} \Phi_{h}\right|_{K}
$$

where $\mathcal{I}$ is the identity. Notice that this choice results in $\tilde{\boldsymbol{E}}_{h}=\tilde{\boldsymbol{E}}_{h}(t, \boldsymbol{x}, \boldsymbol{v})$, due to the $\boldsymbol{v}$ dependence of $\mathcal{H}_{K}$. Then the following total energy is conserved:

$$
\mathscr{E}=\iint_{\Omega} f_{h} \frac{v^{2}}{2} d \boldsymbol{x} d \boldsymbol{v}+\int_{\Omega_{\boldsymbol{x}}} \frac{E_{h}^{2}}{2} d \boldsymbol{x}+\sum_{e_{\boldsymbol{x}} \in \mathcal{E}_{\boldsymbol{x}}} \frac{1}{2} \int_{e_{\boldsymbol{x}}} c_{11} \llbracket \Phi_{h} \rrbracket^{2} d \sigma .
$$

Proof. The proof proceeds as in Theorem 4.1, with some minor modification. Consider the energy balance (4.4) and substitute

$$
\nabla_{\boldsymbol{x}} \Phi_{h}-\tilde{\boldsymbol{E}}_{h}=\mathcal{H}_{K}\left(\nabla_{\boldsymbol{x}} \Phi_{h}-\boldsymbol{E}_{h}\right)
$$


according to (4.10). For a fixed value $\boldsymbol{v}$, the function $\mathcal{H}_{K}(\boldsymbol{x}, \boldsymbol{v}) \boldsymbol{v} f_{h}(\boldsymbol{x}, \boldsymbol{v})$ belongs to $\boldsymbol{R} \boldsymbol{T}_{h}^{k}\left(\mathcal{T}_{\boldsymbol{x}}\right)$, so that we can take $\boldsymbol{z}_{h}=\mathcal{H}_{K} \boldsymbol{v} f_{h}$ in $(3.10)_{1}$ and obtain

$$
\begin{aligned}
\iint_{S_{K_{\boldsymbol{x}}}} f_{h} \boldsymbol{v} \cdot\left(\nabla_{\boldsymbol{x}} \Phi_{h}-\tilde{\boldsymbol{E}}_{h}\right) \mathrm{d} \boldsymbol{x} \mathrm{d} \boldsymbol{v} & =\int_{\Omega_{\boldsymbol{v}}}\left(\int_{K_{\boldsymbol{x}}} \mathcal{H}_{K} \boldsymbol{v} f_{h} \cdot\left(\nabla_{\boldsymbol{x}} \Phi_{h}-\boldsymbol{E}_{h}\right) \mathrm{d} \boldsymbol{x}\right) \mathrm{d} \boldsymbol{v} \\
& =-\int_{\Omega_{\boldsymbol{v}}}\left(\int_{\partial K_{\boldsymbol{x}}} \mathcal{H}_{K} \boldsymbol{v} f_{h} \cdot \boldsymbol{n}\left(\widehat{\Phi}-\Phi_{h}\right) \mathrm{d} \boldsymbol{\sigma}\right) \mathrm{d} \boldsymbol{v} \\
& =\int_{\partial K_{\boldsymbol{x}}} \boldsymbol{n} \cdot\left(\int_{\Omega_{\boldsymbol{v}}} \mathcal{H}_{K} \boldsymbol{v} f_{h}\left(\Phi_{h}-\widehat{\Phi}\right) \mathrm{d} \boldsymbol{v}\right) \mathrm{d} \boldsymbol{\sigma} .
\end{aligned}
$$

Now define

$$
\boldsymbol{q}^{\mathscr{E}}=\left(\widehat{\Phi}-\Phi_{h}\right)\left(\partial_{t} \boldsymbol{E}_{h}-\int_{\Omega_{\boldsymbol{v}}} \mathcal{H}_{K} \boldsymbol{v} f_{h} \mathrm{~d} \boldsymbol{v}\right)+\Phi_{h}\left(\partial_{t} \widehat{\boldsymbol{E}}-\int_{\Omega_{\boldsymbol{v}}} \widehat{\boldsymbol{v} f} \mathrm{~d} \boldsymbol{v}\right)-\int_{\Omega_{\boldsymbol{v}}} \widehat{\boldsymbol{v} f} \frac{v^{2}}{2} \mathrm{~d} \boldsymbol{v}
$$

so that (4.4) can be rewritten as

$$
\frac{d}{d t}\left[\iint_{S_{K_{\boldsymbol{x}}}} f_{h} \frac{v^{2}}{2} \mathrm{~d} \boldsymbol{x} \mathrm{d} \boldsymbol{v}+\int_{K_{\boldsymbol{x}}} \frac{E_{h}^{2}}{2} \mathrm{~d} \boldsymbol{x}\right]=\int_{\partial K_{\boldsymbol{x}}} \boldsymbol{n} \cdot \boldsymbol{q}^{\mathscr{E}} \mathrm{d} \boldsymbol{\sigma} .
$$

The thesis follows from the fact that $\llbracket \boldsymbol{q}^{\mathscr{E}} \rrbracket$ vanishes up to a term which can be included in the definition of the total energy. In fact we have

$$
\begin{aligned}
\llbracket \boldsymbol{q}^{\mathscr{E}} \rrbracket & =\left(\widehat{\Phi}-\left\{\Phi_{h}\right\}\right)\left(\llbracket \partial_{t} \boldsymbol{E}_{h} \rrbracket-\int_{\Omega_{\boldsymbol{v}}} \llbracket \mathcal{H}_{K} \boldsymbol{v} f_{h} \rrbracket \mathrm{d} \boldsymbol{v}\right) \\
& +\llbracket \Phi_{h} \rrbracket \cdot\left(\partial_{t} \widehat{\boldsymbol{E}}-\left\{\partial_{t} \boldsymbol{E}_{h}\right\}-\int_{\Omega_{\boldsymbol{v}}}\left(\widehat{\boldsymbol{v} f}-\left\{\mathcal{H}_{K} \boldsymbol{v} f_{h}\right\}\right) \mathrm{d} \boldsymbol{v}\right) \\
& =-c_{11} \frac{\partial}{\partial t} \frac{\llbracket \Phi_{h} \rrbracket^{2}}{2}-\llbracket \Phi_{h} \rrbracket \cdot \int_{\Omega_{\boldsymbol{v}}}\left(\widehat{\boldsymbol{v f f}}-\left\{\mathcal{H}_{K} \boldsymbol{v} f_{h}\right\}\right) \mathrm{d} \boldsymbol{v} .
\end{aligned}
$$

To see that the last term vanishes, observe that

$$
\llbracket \Phi_{h} \rrbracket=\left(\llbracket \Phi_{h} \rrbracket \cdot \boldsymbol{n}\right) \boldsymbol{n},
$$

so that

$$
\llbracket \Phi_{h} \rrbracket \cdot \int_{\Omega_{\boldsymbol{v}}}\left(\widehat{\boldsymbol{v} f}-\left\{\mathcal{H}_{K} \boldsymbol{v} f_{h}\right\}\right) \mathrm{d} \boldsymbol{v}=\llbracket \Phi_{h} \rrbracket \cdot \boldsymbol{n} \int_{\Omega_{\boldsymbol{v}}} \boldsymbol{n} \cdot\left(\widehat{\boldsymbol{v} f}-\left\{\mathcal{H}_{K} \boldsymbol{v} f_{h}\right\}\right) \mathrm{d} \boldsymbol{v}
$$

and use (3.2), (4.8) and (4.9) to obtain

$$
\begin{aligned}
\boldsymbol{n} \cdot\left\{\mathcal{H}_{K} \boldsymbol{v} f_{h}\right\} & =\boldsymbol{n} \cdot\left\{\mathcal{H}_{K} \boldsymbol{v}\right\}\left\{f_{h}\right\}+\boldsymbol{n} \cdot \frac{1}{4} \llbracket \mathcal{H}_{K} \boldsymbol{v} \rrbracket \llbracket f_{h} \rrbracket \\
& =\boldsymbol{n} \cdot\left\{\boldsymbol{v} f_{h}\right\}+\boldsymbol{n} \cdot \frac{1}{2}|\boldsymbol{v} \cdot \boldsymbol{n}| \llbracket f_{h} \rrbracket
\end{aligned}
$$

We conclude this section with various remarks.

First of all, notice that only the upwind in the "space direction", i.e. on sides $e=\partial K_{\boldsymbol{x}} \times K_{\boldsymbol{v}}$, affects the energy balance and the choice of the electric field $\tilde{\boldsymbol{E}}_{h}$, while the upwinding in the "velocity directions", i.e. on sides $e=K_{\boldsymbol{x}} \times \partial K_{\boldsymbol{v}}$, has no effect on the energy conservation. 
As a second point, it is clear from the proof of Theorem 4.2 that the important step in the closure of the local energy balance is

$$
\iint_{S_{K_{\boldsymbol{x}}}} f_{h} \boldsymbol{v} \cdot\left(\nabla_{\boldsymbol{x}} \Phi_{h}-\tilde{\boldsymbol{E}}_{h}\right) \mathrm{d} \boldsymbol{x} \mathrm{d} \boldsymbol{v}=\int_{\partial K_{\boldsymbol{x}}}\left(\int_{\Omega_{\boldsymbol{v}}} \mathcal{H}_{K} \boldsymbol{v} f_{h} \mathrm{~d} \boldsymbol{v}\right) \cdot \boldsymbol{n}\left(\Phi_{h}-\widehat{\Phi}\right) \mathrm{d} \boldsymbol{\sigma}
$$

and that the choice (4.10) is not necessarily the only possibility. Also, notice that only the boundary values $\left.\mathcal{H}_{K}\right|_{\partial K_{x} \times K_{v}}$ appear in (4.12), so the precise definition of $\mathcal{H}_{K}$ on the element interior is not important.

The third observation concerns the apparently unnatural character of (4.10), in that the electric field depends also on the velocity coordinate. Nevertheless, the fact that such a dependence is necessary in order to close the energy balance can be understood observing the structure of (4.12). In fact, assuming that $\tilde{\boldsymbol{E}}_{h}$ is independent from $\boldsymbol{v}$ leads to an identity where the left-hand-side is proportional to $\int_{\Omega_{v}} f_{h} \boldsymbol{v} \mathrm{d} \boldsymbol{v}$, while the right-hand-side is proportional to $\int_{\Omega_{v}} f_{h} \mathcal{H}_{K} \boldsymbol{v} \mathrm{d} \boldsymbol{v}$. Since these two integrals are independent from each other, and they may even vanish independently, it is hard to imagine being able to satisfy such a condition. The idea of using an electric field which depends explicitly on the velocity coordinate was first introduced in [ACS11], albeit in a different form from the one considered here.

The fourth and last remark concerns the $L^{2}$-norm stability of the resulting scheme. Taking $u_{h}=f_{h}$ in (3.4) yields

$$
\begin{aligned}
\frac{1}{2} \frac{d}{d t} \iint_{K} f_{h}^{2} \mathrm{~d} \boldsymbol{x} \mathrm{d} \boldsymbol{v} & +\int_{\partial_{\boldsymbol{x}} K} \boldsymbol{n} \cdot\left(\widehat{\boldsymbol{v f}}-\frac{1}{2} \boldsymbol{v} f_{h}\right) f_{h} \mathrm{~d} \boldsymbol{\sigma} \mathrm{d} \boldsymbol{v} \\
& -\int_{\partial_{\boldsymbol{v}} K} \boldsymbol{n} \cdot\left(\widehat{\boldsymbol{E f}}-\frac{1}{2} \tilde{\boldsymbol{E}}_{h} f_{h}\right) f_{h} \mathrm{~d} \boldsymbol{x} \mathrm{d} \boldsymbol{\tau}=0
\end{aligned}
$$

and, after summing over all elements $K$,

$$
\begin{aligned}
\frac{1}{2} \frac{d}{d t} \iint_{\Omega} f_{h}^{2} \mathrm{~d} \boldsymbol{x} \mathrm{d} \boldsymbol{v}+\sum_{e_{\boldsymbol{v}, \boldsymbol{x}}, e_{\boldsymbol{x}, \boldsymbol{v}} \in \mathcal{E}} & {\left[\int_{e_{\boldsymbol{v}, \boldsymbol{x}}}\left(\widehat{\boldsymbol{v} f} \cdot \llbracket f_{h} \rrbracket-\frac{1}{2} \llbracket \boldsymbol{v} f_{h}^{2} \rrbracket\right) \mathrm{d} \boldsymbol{\sigma} \mathrm{d} \boldsymbol{v}\right.} \\
& \left.-\int_{e_{\boldsymbol{x}, \boldsymbol{v}}}\left(\widehat{\boldsymbol{E} f} \cdot \llbracket f_{h} \rrbracket-\frac{1}{2} \llbracket \tilde{\boldsymbol{E}}_{h} f_{h}^{2} \rrbracket\right) \mathrm{d} \boldsymbol{x} \mathrm{d} \boldsymbol{\tau}\right]=0 .
\end{aligned}
$$

Concerning the first term, substituting the weighted upwind flux (3.7) we have

$$
\widehat{\boldsymbol{v} f} \cdot \llbracket f_{h} \rrbracket-\frac{1}{2} \llbracket \boldsymbol{v} f_{h}^{2} \rrbracket=\frac{1}{2} \omega|\boldsymbol{v} \cdot \boldsymbol{n}| \llbracket f_{h} \rrbracket^{2} \geq 0 .
$$

Concerning the second term, using (3.2) and (3.3) we have

$$
-\widehat{\boldsymbol{E f}} \cdot \llbracket f_{h} \rrbracket+\frac{1}{2} \llbracket \tilde{\boldsymbol{E}}_{h} f_{h}^{2} \rrbracket=\frac{1}{2} \omega\left|\tilde{\boldsymbol{E}}_{h} \cdot \boldsymbol{n}\right| \llbracket f_{h} \rrbracket^{2}+\frac{1}{2} \llbracket \tilde{\boldsymbol{E}}_{h} \rrbracket\left(\left\{f_{h}\right\}^{2}-\frac{1}{4} \llbracket f_{h} \rrbracket^{2}\right) .
$$

Now, if $\tilde{\boldsymbol{E}}_{h}$ is independent from $\boldsymbol{v}$, its jump on $e_{\boldsymbol{x}, \boldsymbol{v}}$ vanishes and $\left\|f_{h}\right\|_{L^{2}(\Omega)}$ turns out to be a nonincreasing quantity. However, when a dependence of $\tilde{\boldsymbol{E}}_{h}$ is introduced, as in the energy conserving method (4.10), the sign of the last term is unknown, and nothing can be said about the time evolution of $\left\|f_{h}\right\|_{L^{2}(\Omega)}$. Since, as discussed above, a $\boldsymbol{v}$ dependence for $\tilde{\boldsymbol{E}}_{h}$ seems to be required in order to ensure energy conservation 
with upwind fluxes, this result seems to indicate a contradiction between discrete energy conservation and nonincreasing $\left\|f_{h}\right\|_{L^{2}(\Omega)}$ for the upwind method. Concerning the practical impact of this remark, we anticipate that all our numerical computations employing (4.10) and upwind fluxes show the desired, nonincreasing behaviour of $\left\|f_{h}\right\|_{L^{2}(\Omega)}$, suggesting that, for these specific cases, the terms proportional to $\omega$ dominate the term proportional to $\llbracket \tilde{\boldsymbol{E}}_{h} \rrbracket$.

5. Spectral element DG discretization of the Vlasov-Poisson system. To obtain the spectral element formulation corresponding to (3.4), (3.10) and (3.11), one simply makes the formal substitution

$$
\int_{.} \mapsto \int_{\cdot, w}
$$

introducing the quadrature formulae defined in $\S$ 3.1. The resulting scheme can be analyzed within the framework of the generalized Galerkin methods, which is discussed in [CHQZ07], chapter 5. We provide now the details for the energy conserving formulation addressed in Theorem 4.2, since all the other cases can be easily deduced from this one.

We consider the Poisson problem (3.10) first. Introducing the numerical quadrature formulae yields the problem:

find $\left(\boldsymbol{E}_{h}, \Phi_{h}\right) \in \boldsymbol{R} \boldsymbol{T}_{h}^{k}\left(\mathcal{T}_{\boldsymbol{x}}\right) \times V_{h}^{k}\left(\mathcal{T}_{\boldsymbol{x}}\right)$ such that, for any $\left(\boldsymbol{z}_{h}, p_{h}\right) \in \boldsymbol{R} \boldsymbol{T}_{h}^{k}\left(\mathcal{T}_{\boldsymbol{x}}\right) \times V_{h}^{k}\left(\mathcal{T}_{\boldsymbol{x}}\right)$ and any $K_{\boldsymbol{x}} \in \mathcal{T}_{\boldsymbol{x}}$,

$$
\begin{array}{r}
\int_{K_{\boldsymbol{x}}, \boldsymbol{w}_{+}} \boldsymbol{E}_{h} \cdot \boldsymbol{z}_{h} \mathrm{~d} \boldsymbol{x}-\int_{K_{\boldsymbol{x}}, \boldsymbol{w}} \nabla_{\boldsymbol{x}} \Phi_{h} \cdot \boldsymbol{z}_{h} \mathrm{~d} \boldsymbol{x}-\int_{\partial K_{\boldsymbol{x}}, w}\left(\widehat{\Phi}-\Phi_{h}\right) \boldsymbol{n} \cdot \boldsymbol{z}_{h} \mathrm{~d} \boldsymbol{\sigma}=0 \\
\int_{K_{\boldsymbol{x}}, \boldsymbol{w}} \boldsymbol{E}_{h} \cdot \nabla_{\boldsymbol{x}} p_{h} \mathrm{~d} \boldsymbol{x}-\int_{\partial K_{\boldsymbol{x}}, w} \boldsymbol{n} \cdot \widehat{\boldsymbol{E}} p_{h} \mathrm{~d} \boldsymbol{\sigma}=-\int_{K_{\boldsymbol{x}}, w}\left(1-\rho_{h}\right) p_{h} \mathrm{~d} \boldsymbol{x}
\end{array}
$$

where, for two vector valued functions $\boldsymbol{r}_{h}, \boldsymbol{s}_{h}$, we use the notation

$$
\int_{K_{\boldsymbol{x}}, \boldsymbol{w}_{+}} \boldsymbol{r}_{h} \cdot \boldsymbol{s}_{h} \mathrm{~d} \boldsymbol{x}=\sum_{\alpha=1}^{d} \int_{K_{\boldsymbol{x}}, w_{+}^{\alpha}} r_{h}^{\alpha} s_{h}^{\alpha} \mathrm{d} \boldsymbol{x}
$$

and

$$
\int_{K_{\boldsymbol{x}}, \boldsymbol{w}} \boldsymbol{r}_{h} \cdot \boldsymbol{s}_{h} \mathrm{~d} \boldsymbol{x}=\sum_{\alpha=1}^{d} \int_{K_{\boldsymbol{x}}, w} r_{h}^{\alpha} s_{h}^{\alpha} \mathrm{d} \boldsymbol{x}
$$

Notice that the second term in $(5.1)_{1}$ and the first one in $(5.1)_{2}$ require the interpolation of $\boldsymbol{z}_{h}$ and $\boldsymbol{E}_{h}$ on points which are different from the nodes of the Lagrangian basis of the corresponding space $\boldsymbol{R} \boldsymbol{T}_{h}^{k}\left(\mathcal{T}_{\boldsymbol{x}}\right)$, while for all the remaining terms the quadrature nodes coincide with the nodes of the Lagrangian basis. The required interpolation, anyway, turns out to be one-dimensional, so that it does not result in a significant overhead.

We then consider the definition of $\tilde{\boldsymbol{E}}_{h}$, equation (4.10). The following definition allows to recover Theorem 4.2 even in the spectral element case: let $\tilde{\boldsymbol{E}}_{h} \in \boldsymbol{W}_{h}^{k}\left(\mathcal{T}_{\boldsymbol{x}}\right)$ be defined by

$$
\int_{K_{\boldsymbol{x}}, \boldsymbol{w}} \tilde{\boldsymbol{E}}_{h} \cdot \boldsymbol{z}_{h} \mathrm{~d} \boldsymbol{x}=\int_{K_{\boldsymbol{x}}, \boldsymbol{w}_{+}} \boldsymbol{E}_{h} \cdot \mathcal{H}_{K} \boldsymbol{z}_{h} \mathrm{~d} \boldsymbol{x}+\int_{K_{\boldsymbol{x}}, \boldsymbol{w}} \nabla_{\boldsymbol{x}} \Phi_{h} \cdot\left(\mathcal{I}-\mathcal{H}_{K}\right) \boldsymbol{z}_{h} \mathrm{~d} \boldsymbol{x}
$$


for any $\boldsymbol{z}_{h} \in \boldsymbol{W}_{h}^{k}\left(\mathcal{T}_{\boldsymbol{x}}\right)$ and any $K_{\boldsymbol{x}} \in \mathcal{T}_{\boldsymbol{x}}$.

Finally, concerning the Vlasov equation (3.4), we have: find $f_{h} \in V_{h}^{k}\left(\mathcal{T}_{\boldsymbol{x}}\right)$ such that, for any $u_{h} \in V_{h}^{k}\left(\mathcal{T}_{\boldsymbol{x}}\right)$ and any $K \in \mathcal{T}$,

$$
\begin{array}{r}
\iint_{K, w} \partial_{t} f_{h} u_{h} \mathrm{~d} \boldsymbol{x} \mathrm{d} \boldsymbol{v}-\iint_{K, \boldsymbol{w}} \boldsymbol{v} f_{h} \cdot \nabla_{\boldsymbol{x}} u_{h} \mathrm{~d} \boldsymbol{x} \mathrm{d} \boldsymbol{v}+\int_{\partial_{\boldsymbol{x}} K, w} \boldsymbol{n} \cdot \widehat{\boldsymbol{v f f}} u_{h} \mathrm{~d} \boldsymbol{\sigma} \mathrm{d} \boldsymbol{v} \\
+\iint_{K, \boldsymbol{w}} \tilde{\boldsymbol{E}}_{h} f_{h} \cdot \nabla_{\boldsymbol{v}} u_{h} \mathrm{~d} \boldsymbol{x} \mathrm{d} \boldsymbol{v}-\int_{\partial_{\boldsymbol{v}} K, w} \boldsymbol{n} \cdot \widehat{\boldsymbol{E} f} u_{h} \mathrm{~d} \boldsymbol{x} \mathrm{d} \boldsymbol{\tau}=0 .
\end{array}
$$

Notice that for all the integrals appearing in (5.3) the quadrature nodes coincide with the nodes of the Lagrangian basis, so that no interpolation is required.

As anticipated, it is easy to verify that all the steps of the proof of Theorem 4.2 can be repeated for the system (5.1), (5.2) and (5.3), so that the following energy is conserved:

$$
\mathscr{E}_{w}=\iint_{\Omega, w} f_{h} \frac{v^{2}}{2} \mathrm{~d} \boldsymbol{x} \mathrm{d} \boldsymbol{v}+\int_{\Omega_{x}, \boldsymbol{w}_{+}} \frac{E_{h}^{2}}{2} \mathrm{~d} \boldsymbol{x}+\sum_{e_{\boldsymbol{x}} \in \mathcal{E}_{\boldsymbol{x}}} \frac{1}{2} \int_{e_{\boldsymbol{x}}, w} c_{11} \llbracket \Phi_{h} \rrbracket^{2} \mathrm{~d} \boldsymbol{\sigma} .
$$

The main advantage of the spectral element formulation compared to the standard method, using exact integration, is its higher efficiency, resulting from the collocation of the quadrature nodes and the degrees of freedom of the Lagrangian basis. This can be understood observing that: a) the mass matrices are diagonal, b) the computation of the directional derivatives at a given quadrature point involves only a limited subset of the degrees of freedom of each element, and c) the computation of the numerical fluxes and the corresponding boundary integrals involves only few degrees of freedom per each element.

We now briefly illustrate points b) and c) in the case $d=1$. In Figure 5.1, left, the LGL points for $k=3$ and for an element $K \in \mathcal{T}$ in a two-dimensional phase space are shown with "bullets". According to the definition of the Lagrangian basis and of the quadrature rule given in $\S 3.1$, these points serve both as degrees of freedom and quadrature nodes. In the same figure, the LGL points defined on each side of the element are also displayed with "stars". Since the LGL points always include the boundaries of the quadrature integrals, the "stars" are superimposed to some of the "bullets". To evaluate the volume integrals appearing in the DG-SE
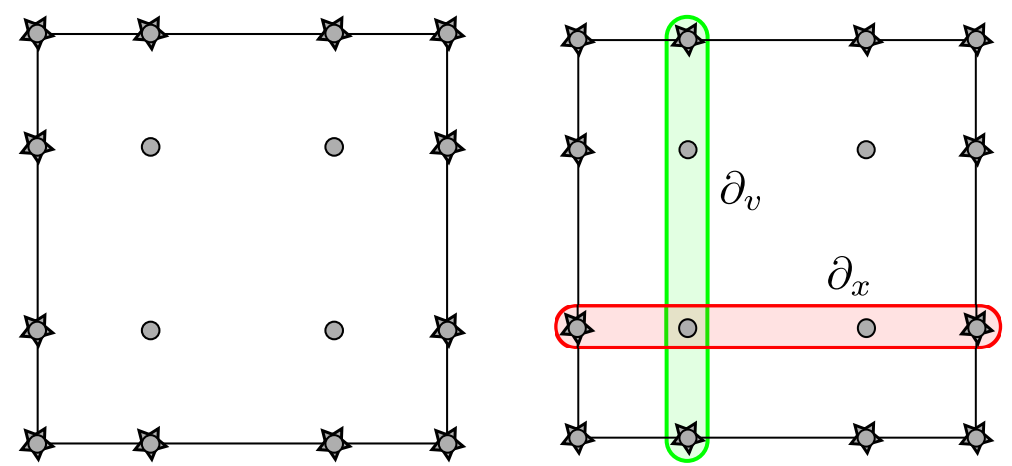

FIG. 5.1. LGL points for a two-dimensional element, $k=3$ (left) and corresponding stencils for the computation of the $x$ and $v$ partial derivatives (right).

formulation, we need to sample the various fields at the internal quadrature points, 
together with the corresponding derivatives. Sampling the fields at an LGL point is straightforward, since it amounts to accessing the collocated degree of freedom. Sampling the derivatives can be done in terms of few degrees of freedom: for instance, considering Figure 5.1, right, to compute $\partial_{\boldsymbol{x}} f_{h}$ at the bottom left, internal node, only the four degrees of freedom highlighted in red are required. Similarly, the computation of $\partial_{v} f_{h}$ at the same point requires the four degrees of freedom highlighted in green. To evaluate the boundary integrals, the numerical fluxes are required at the boundary quadrature points, marked with a star, which requires sampling the various fields at these points. Thanks to the fact that the "stars" are superimposed to the "bullets", this operation reduces again to access a limited number of local degrees of freedom.

6. Time discretization. Equation (5.3) is an ordinary differential equation which can be written as

$$
\mathbf{f}^{\prime}=\mathbf{R}(\mathbf{f})
$$

where $\mathbf{f}$ is the array of the nodal values of the distribution function $f_{h}$ and $\mathbf{R}$ denotes the right-hand-side; (6.1) can be discretized in time using a time integrator method, following the classical approach of the method of lines. The standard choice is represented by Runge-Kutta type methods, and we consider here the classical RK4 method. Hence, introducing a time step $\Delta t$ and time levels $t^{n}=n \Delta t$, for $n=0, \ldots, T / \Delta t$, to advance the numerical solution from $\mathbf{f}^{n}=\mathbf{f}\left(t^{n}\right)$ to $\mathbf{f}^{n+1}$ we have to solve

$$
\begin{array}{ll}
\mathbf{K}_{1}=\mathbf{R}\left(\mathbf{f}^{n}\right) & \mathbf{K}_{2}=\mathbf{R}\left(\mathbf{f}^{n}+\frac{\Delta t}{2} \mathbf{K}_{1}\right) \\
\mathbf{K}_{3}=\mathbf{R}\left(\mathbf{f}^{n}+\frac{\Delta t}{2} \mathbf{K}_{2}\right) & \mathbf{K}_{4}=\mathbf{R}\left(\mathbf{f}^{n}+\Delta t \mathbf{K}_{3}\right) \\
\mathbf{f}^{n+1}=\mathbf{f}^{n}+\frac{\Delta t}{6}\left(\mathbf{K}_{1}+2 \mathbf{K}_{2}+2 \mathbf{K}_{3}+\mathbf{K}_{4}\right) .
\end{array}
$$

At each stage of (6.2), the computation of $\mathbf{R}(\mathbf{f})$ requires the solution of the Poisson problem (5.1).

Notice that the energy balances (4.6) and (4.11) are derived using the product rule for the exact time derivative, which does not hold for the time integrator (6.2). Hence, an error is introduced in the energy balance proportional to the time discretization error. This error is quantified numerically in $\S 7$. This error could be avoided using a centered scheme as discussed in [Kra13], but this would lead to an implicit formulation.

The time integrator (6.2), albeit representing and adequate choice for our problem, could be less than optimal concerning the computational efficiency. In fact, in order to compute stable numerical simulations, the time-step must be typically smaller than what would be desirable in terms of a trade-off between accuracy and efficiency. There are at least three possibility to tackle this issue: semi-Lagrangian methods [SRBG99, QS11], implicit time integrators, and exponential time integrators. Each of these methods has merits and drawbacks; here we would like to explore the use of exponential time integrators as an alternative to RK ones.

The idea of integrating exactly the linear part of an ODE, thus obtaining an exponential integrator, in order to improve both the accuracy and the stability of the scheme, has been first considered in [Law67]. A number of methods have then been proposed and analyzed, for both scalar equations and systems. Here, we follow the presentation of [SSS09], where the interested reader can also find a more comprehensive bibliography. The first step in the construction of an exponential time integrator is rewriting a generic time-step of (6.1) as

$$
\begin{aligned}
\mathbf{f}^{\prime} & =\mathcal{J}_{\mathbf{R}}^{n}\left(\mathbf{f}-\mathbf{f}^{n}\right)+\mathcal{O}\left(\left(\mathbf{f}-\mathbf{f}^{n}\right)^{2}\right), \quad t \in\left(t^{n}, t^{n+1}\right) \\
\mathbf{f}\left(t^{n}\right) & =\mathbf{f}^{n}
\end{aligned}
$$


where $\mathcal{J}_{\mathbf{R}}^{n}$ is the Jacobian of the right-hand-side evaluated at $\mathbf{f}^{n}$. Discarding the higher order term, (6.3) reduces to a linear equation, which can be integrated exactly obtaining

$$
\mathbf{f}^{n+1}=\mathbf{f}^{n}+\Delta t \phi\left(\Delta t \mathcal{J}_{\mathbf{R}}^{n}\right) \mathbf{R}\left(\mathbf{f}^{n}\right)
$$

where the function $\phi$ is defined as

$$
\phi(z)=\frac{\mathrm{e}^{z}-1}{z}
$$

for a scalar argument $z \in \mathbb{C}$ and is extended for matrix arguments by power series (see also [GvL12]). Method (6.4) is the simplest exponential integrator that can be constructed; thanks to the presence of the Jacobian matrix, it is a second order method, which is referred to as Exponential Rosenbrock of second order (ERB2). Before this method can be used to solve large systems of differential equations, however, two nontrivial problems must still be addressed, namely the computation of the Jacobian matrix and the computation of $\phi\left(\Delta t \mathcal{J}_{\mathbf{R}}^{n}\right)$. Many approaches have been discussed to compute the exponential of a generic matrix $\mathcal{A}$, or equivalently the quantity $\phi(\mathcal{A})$, and a review can be found in [MvL06]; in this work, we consider the following two alternatives: Krylov space iterations (see [Saa91, HLS98]) and interpolation at real Leja points (see [CVB04, CO09]). Both these techniques are iterative methods, and each iteration requires the computation of a matrix-vector product of the form $\mathcal{A} \mathbf{v}$, for suitable vectors $\mathbf{v}$. This has some important consequences.

First of all, the fact that only the action of $\mathcal{A}$ is required, and not the explicit knowledge of the matrix itself, provides a convenient solution for the problem of computing the Jacobian matrix. In fact, since we are interested in the case $\mathcal{A}=\Delta t \mathcal{J}_{\mathbf{R}}^{n}$, we can consider the approximation

$$
\mathcal{J}_{\mathbf{R}}^{n} \mathbf{v} \approx \frac{1}{\varepsilon_{\mathrm{diff}}}\left(\mathbf{R}\left(\mathbf{f}^{n}+\varepsilon_{\mathrm{diff}} \mathbf{v}\right)-\mathbf{R}\left(\mathbf{f}^{n}\right)\right),
$$

where $\varepsilon_{\text {diff }}$ is a small parameter. Notice that a centered finite difference could be also considered, although at the price of doubling the evaluations of $\mathbf{R}(\mathbf{f})$; this solution has been considered in [GBNS14].

A second consequence is that a stopping criterion must be provided: a large number of iterations results in an accurate approximation of $\phi\left(\Delta t \mathcal{J}_{\mathbf{R}}^{n}\right)$, but also leads to a higher computational cost. For both methods, an adaptive stopping criterion is available, based on an estimate of the error in the approximation of $\phi\left(\Delta t \mathcal{J}_{\mathbf{R}}^{n}\right)$. For the Krylov space iterations, we follow [SSS09], section 2.2, and stop the Krylov iterations when

$$
\left.\| \phi\left(\Delta t \mathcal{J}_{\mathbf{R}}^{n}\right)-\phi \widetilde{\left(\Delta t \mathcal{J}_{\mathbf{R}}^{n}\right.}\right) \|_{2} \leq \varepsilon_{\mathrm{Kry}}
$$

where a tilde denotes the numerical approximation and $\varepsilon_{\mathrm{Kry}}$ is a prescribed tolerance, while for the Leja point interpolation method new terms are introduced in the Newton polynomial interpolation as far as they are larger than $\varepsilon_{\text {Lej }}\left\|\phi \widetilde{\left(\Delta t \mathcal{J}_{\mathbf{R}}^{n}\right)}\right\|_{2}$. Concerning the computational cost, by virtue of (6.5), each iteration requires one additional evaluation of the right-hand-side, and thus a computational cost analogous to a stage of (6.2). A reasonable comparison of the computational cost of each time step of RK4 and ERB2 can thus be made in terms of the efficiency index

$$
\eta_{\mathrm{eff}}=\frac{4}{\Delta t^{\mathrm{RK} 4}} \frac{\Delta t^{\mathrm{ERB} 2}}{n_{\mathrm{rhs}}}
$$


where $n_{\text {rhs }}$ is the number of evaluations of the right-hand-side $\mathbf{R}(\mathbf{f})$ in the time step, namely 1 plus the number of evaluations of $\mathcal{J}_{\mathbf{R}}^{n} \mathbf{v}$ in (6.5), for either the Krylov space iterations or the interpolation at the Leja points.

The last consequence of the iterative nature of the computation of $\phi\left(\Delta t \mathcal{J}_{\mathbf{R}}^{n}\right)$ is that the convergence rate of the methods depends on the spectral properties of the matrix $\mathcal{J}_{\mathbf{R}}^{n}$. Some details concerning this aspect are collected in Appendix A.

7. Numerical validation. In this section we address the numerical validation of the spectral element DG formulation. The various test cases are selected to assess the performance of the method concerning the following aspects: convergence rates, overall ability to represent relevant physical phenomena, robustness and discrete conservation properties. References for the considered test cases can be found in [CK76, Sho79, NY99, CMS10, HGMM12, MSM+13, CGM13]. For $§ 7.1, \S 7.2$ and $\S 7.3$, the emphasis is on the spatial discretization, while for the time integration we consider the RK4 scheme with appropriately small time-step. The effect of the time discretization is considered in $\S 7.4$, where the RK4 scheme is compared with the exponential time integrators discussed in $\S 6$.

For most of the test cases, the electrostatic energy is an important diagnostic. To ensure consistency with the numerical discretization, for $\boldsymbol{E}_{h} \in \boldsymbol{R} \boldsymbol{T}_{h}^{k}\left(\mathcal{T}_{\boldsymbol{x}}\right)$ we compute the electrostatic energy as

$$
\mathscr{E}_{w}^{e \mathrm{el}}=\int_{\Omega_{\boldsymbol{x}}, \boldsymbol{w}_{+}} \frac{E_{h}^{2}}{2} \mathrm{~d} \boldsymbol{x}+\sum_{e_{\boldsymbol{x}} \in \mathcal{E}_{\boldsymbol{x}}} \frac{1}{2} \int_{e_{\boldsymbol{x}}, w} c_{11} \llbracket \Phi_{h} \rrbracket^{2},
$$

alongside with the kinetic energy

$$
\stackrel{\mathscr{E} \text { kin }}{w}=\iint_{\Omega, w} f_{h} \frac{v^{2}}{2} \mathrm{~d} \boldsymbol{x} \mathrm{d} \boldsymbol{v}
$$

and the total energy defined by (5.4). When $\boldsymbol{E}_{h} \in \boldsymbol{W}_{h}^{k}\left(\mathcal{T}_{\boldsymbol{x}}\right)$, the same formula is used with the substitution $\int_{\Omega_{x}, \boldsymbol{w}_{+}} \mapsto \int_{\Omega_{\boldsymbol{x}}, \boldsymbol{w}}$. For ease of comparison with other references, we often plot the square root $\sqrt{\mathscr{E}_{w}^{\mathrm{el}}}$.

Given that in all the formulations considered here we take $f_{h} \in V_{h}^{k}(\mathcal{T}), \Phi_{h} \in$ $V_{h}^{k}\left(\mathcal{T}_{\boldsymbol{x}}\right)$ and the elliptic numerical fluxes (3.12), we then distinguish among the following formulations:

- centered method $(\mathrm{CM})$ : with $\boldsymbol{E}_{h} \in \boldsymbol{W}_{h}^{k}\left(\mathcal{T}_{\boldsymbol{x}}\right), \tilde{\boldsymbol{E}}_{h}=\boldsymbol{E}_{h}$, according to (4.5), and centered numerical fluxes in (3.5) and (3.6);

- space centered, velocity upwind method (SCVU): with $\boldsymbol{E}_{h} \in \boldsymbol{W}_{h}^{k}\left(\mathcal{T}_{\boldsymbol{x}}\right), \tilde{\boldsymbol{E}}_{h}=$ $\boldsymbol{E}_{h}$ and centered and upwind numerical fluxes in (3.5) and (3.6), respectively;

- upwind method (UM): with $\boldsymbol{E}_{h} \in \boldsymbol{W}_{h}^{k}\left(\mathcal{T}_{\boldsymbol{x}}\right), \tilde{\boldsymbol{E}}_{h}=\boldsymbol{E}_{h}$ and upwind numerical fluxes in (3.5) and (3.6);

- upwind, energy conserving method (UECM): with $\boldsymbol{E}_{h} \in \boldsymbol{R} \boldsymbol{T}_{h}^{k}\left(\mathcal{T}_{\boldsymbol{x}}\right), \tilde{\boldsymbol{E}}_{h}$ defined by (4.10) and upwind numerical fluxes in (3.5) and (3.6).

7.1. Convergence rate of the electric field. We consider in this section the accuracy of the reconstruction of the electric field $\tilde{\boldsymbol{E}}_{h}$ for different choices of the finite element spaces and the stabilization coefficient. For this purpose, we solve (3.9) in two dimensions with

$$
\rho\left(x^{1}, x^{2}\right)=\frac{1}{2} \pi^{2} \cos \left(\frac{\pi}{2} x^{1}\right) \cos \left(\frac{\pi}{2} x^{2}\right)+1
$$


TABLE 7.1

$L^{2}$ error norms for the three considered reconstructions of the electric field for problem (7.3) for polynomial degree $k=4$ and $k=5$, with the corresponding numerical convergence rates. The results are obtained considering uniform grids with $16 n^{-2}$ elements for increasing $n$.

\begin{tabular}{|c||c|c||c|c||c|c||}
\hline \multicolumn{2}{|c||}{$k=4$} \\
\hline $\log _{2} n$ & $\left\|\nabla_{\boldsymbol{x}} \Phi_{e x}-\boldsymbol{E}_{h, \mathrm{LDG}}\right\|_{L^{2}}$ & $\left\|\nabla_{\boldsymbol{x}} \Phi_{e x}-\tilde{\boldsymbol{E}}_{h, h-1}\right\|_{L^{2}}$ & \multicolumn{2}{|||}{$\left\|\nabla_{\boldsymbol{x}} \Phi_{e x}-\tilde{\boldsymbol{E}}_{h, 0}\right\|_{L^{2}}$} \\
\hline 1 & $1.6 \cdot 10^{-1}$ & - & $1.6 \cdot 10^{-1}$ & - & $1.6 \cdot 10^{-1}$ & - \\
\hline 2 & $7.2 \cdot 10^{-3}$ & 4.485 & $7.4 \cdot 10^{-3}$ & 4.443 & $7.9 \cdot 10^{-3}$ & 4.356 \\
\hline 3 & $4.4 \cdot 10^{-4}$ & 4.042 & $4.6 \cdot 10^{-4}$ & 4.026 & $4.6 \cdot 10^{-4}$ & 4.094 \\
\hline 4 & $2.7 \cdot 10^{-5}$ & 4.013 & $2.8 \cdot 10^{-5}$ & 4.008 & $2.8 \cdot 10^{-5}$ & 4.029 \\
\hline \hline \multicolumn{7}{|c||}{$k=5$} \\
\hline $\log _{2} n$ & $\left\|\nabla_{\boldsymbol{x}} \Phi_{e x}-\boldsymbol{E}_{h, \mathrm{LDG}}\right\|_{L^{2}}$ & $\left\|\nabla_{\boldsymbol{x}} \Phi_{e x}-\tilde{\boldsymbol{E}}_{h, h}-1\right\|_{L^{2}}$ & $\left\|\nabla_{\boldsymbol{x}} \Phi_{e x}-\tilde{\boldsymbol{E}}_{h, 0}\right\|_{L^{2}}$ \\
\hline 1 & $6.0 \cdot 10^{-3}$ & - & $6.0 \cdot 10^{-3}$ & - & $6.0 \cdot 10^{-3}$ & - \\
\hline 2 & $5.8 \cdot 10^{-4}$ & 3.358 & $5.9 \cdot 10^{-4}$ & 3.336 & $6.0 \cdot 10^{-4}$ & 3.331 \\
\hline 3 & $1.9 \cdot 10^{-5}$ & 4.925 & $1.9 \cdot 10^{-5}$ & 4.940 & $1.9 \cdot 10^{-5}$ & 4.936 \\
\hline 4 & $6.1 \cdot 10^{-7}$ & 4.978 & $6.1 \cdot 10^{-7}$ & 4.983 & $6.1 \cdot 10^{-7}$ & 4.989 \\
\hline
\end{tabular}

and $\Omega_{\boldsymbol{x}}=(-2,2)^{2}$, with solution

$$
\Phi_{e x}=\cos \left(\frac{\pi}{2} x^{1}\right) \cos \left(\frac{\pi}{2} x^{2}\right) .
$$

We consider the $L^{2}$ error for the following definitions of the reconstructed electric field: $\boldsymbol{E}_{h, \text { LDG }}$ is the solution of (3.10) with $\boldsymbol{E}_{h} \in \boldsymbol{W}_{h}^{k}\left(\mathcal{T}_{\boldsymbol{x}}\right)$ and $c_{11}=h^{-1}(k+1)^{2}$, $\tilde{\boldsymbol{E}}_{h, h^{-1}}$ is the field obtained with the reconstruction (4.10) and $c_{11}=h^{-1}(k+1)^{2}$ and $\tilde{\boldsymbol{E}}_{h, 0}$ is the field obtained again with the reconstruction (4.10) but now setting $c_{11}=0$ (for these last two reconstructions we consider the values corresponding to $v^{\alpha} \geq 0$ in (4.7), however similar results are obtained for different combinations of $\left.v^{\alpha}\right)$. The results are shown in Table 7.1 for the two cases $k=4$ and $k=5$; it can be seen that all the reconstructions display the optimal convergence rate, and that the values of the errors are comparable. Other results, not included here, suggest that the fact that the asymptotic convergence rate is approached from above for even $k$ and from below for odd $k$ is a general feature.

7.2. One-dimensional test cases. The first test case considered here is the standard Landau damping problem, using the setup described in [CMS10, HGMM12, $\mathrm{MSM}^{+} 13$, CGM13], which we briefly recall for the sake of completeness. The computational domain is $\Omega_{\boldsymbol{x}}=(0,4 \pi)$ and $\Omega_{\boldsymbol{v}}=(-10,10)$ and the initial condition is

$$
f_{0}(x, v)=\frac{1}{\sqrt{2 \pi}} \mathrm{e}^{-\frac{v^{2}}{2}}(1+A \sin (\kappa x)),
$$

with $\kappa=0.5$. The parameter $A$ can be chosen in order to obtain either a linear or a nonlinear problem: we consider here $A=0.01$, resulting in a linear regime, and $A=0.5$, corresponding to a nonlinear one.

Concerning the linear regime, the time evolution of $\sqrt{\mathscr{E}_{w}^{e}}$ is represented in Figure 7.1 for the UECM with $c_{11}=0$ and polynomial degree $k=6$, for various phase space resolutions. The time-step is $\Delta t=6.25 \cdot 10^{-4}$ for all the computations, so that the error results essentially from the space discretization. For this problem, the dominant mode of the linear solution is characterized by the damping constant $\gamma=-0.1534$ and the angular frequency $\omega=1.416$. It can be observed that all the computations are in good agreement with the linear solution until a discrete recurrence appears. 

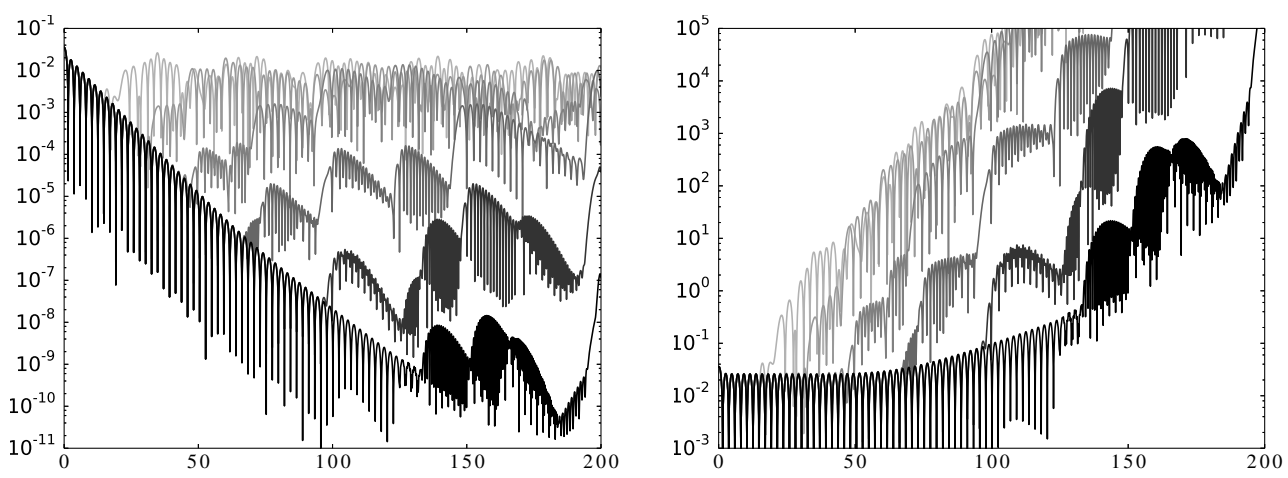

FIG. 7.1. Linear Landau damping, time evolution of the electrostatic energy for $k=6$ and grid resolution, from light gray to black: $5 \times 10$ elements in space and velocity, respectively, $10 \times 20$ elements, $20 \times 40$ elements, $40 \times 80$ elements, $80 \times 160$ elements and $160 \times 320$ elements. The left plot shows $\sqrt{\mathscr{E}_{w}^{\mathrm{el}}}$, while the right plot shows $\exp (0.1534 t) \sqrt{\mathscr{E}_{w}^{\mathrm{el}}}$.

To simplify the comparison with the linear solution, we plot in Figure 7.1, right, the quantity $\exp (0.1534 t) \sqrt{\mathscr{E}_{w}^{e l}}$, which should exhibit oscillations with constant amplitude. Here, it can be observed that the computed electric energy in fact does show constant amplitude oscillations up to $t \approx 50$, provided a sufficient resolution is used, while for larger times the amplitude increases (i.e., the numerical solution decays at a lower rate compared with the linear solution). This effect, which can be observed also in $\left[\mathrm{MSM}^{+} 13\right]$, is independent from the resolution and is due to nonlinear effects; additional computations with $A=0.001$, not reported here, are in agreement with the linear solution for longer times. The LGL points for $k=6$ are

$$
\left\{\hat{\xi}_{i}^{1 \mathrm{D}, 6}, \quad i=0, \ldots, 6\right\}=\{-1.00,-0.83,-0.47,0.00,0.47,0.83,1.00\} .
$$

These points, together with the associated weights, define a local grid within each element with nonuniform spacing $\Delta v$, which is responsible for the various recurrence times observed in the electric energy, according to the analysis of [CK76]. A more detailed analysis is presented in [CGM13]; here, we mention that our results are qualitatively similar to those of this last reference as well as [AS12]. From our results, it seems that the nonuniform distribution of the LGL points anticipates the recursion: a uniform grid with the same number of degrees of freedom as the spectral element, DG formulation with $k=6$ would have $\Delta v \approx \frac{1}{7}\left|K_{\boldsymbol{v}}\right|$, resulting in a recurrence time $\tau^{\text {rec }}=$ $\frac{2 \pi}{\kappa \Delta v}$, while our results suggest that the electric energy reaches a level comparable to the initial condition after a time which is consistent with a spacing $\Delta v \approx \frac{1}{4}\left|K_{\boldsymbol{v}}\right|$. Since the clustering of the LGL points toward the element boundaries becomes more pronounced for increasing polynomial degree $k$, this could provide an upper limit for $k$ for practical computations.

Concerning the nonlinear Landau damping problem, we consider the time evolution of the electrostatic energy, the effect of the numerical dissipation and the positivity of the distribution function for the UECM with $c_{11}=0$ and different combinations of polynomial degree and grid resolution. More specifically, we consider the low resolution cases: $k=4$ and $56 \times 112$ elements in space and velocity, $k=5$ and $47 \times 94$ elements, $k=6$ and $40 \times 80$ elements, $k=7$ and $35 \times 70$ elements; high resolution cases are then obtained, for the same polynomial degrees, by doubling the number of elements in space and velocity. The number of degrees of freedom is approximately 
the same among the low resolution cases as well as among the high resolution ones. For all the computations, the time-step is $\Delta t=6.25 \cdot 10^{-4}$. The evolution of the electrostatic energy for the first four modes, defined as (see [HGMM12])

$$
\mathscr{E}_{w, n}^{\mathrm{el}}=\frac{\kappa}{2 \pi}\left(E_{s, n}^{2}+E_{c, n}^{2}\right)
$$

with

$$
E_{s, n}=\int_{\Omega_{\boldsymbol{x}}, \boldsymbol{w}_{+}} E_{h} \sin (n \kappa x) \mathrm{d} x, \quad E_{c, n}=\int_{\Omega_{\boldsymbol{x}}, \boldsymbol{w}_{+}} E_{h} \cos (n \kappa x) \mathrm{d} x,
$$

is displayed in Figure 7.2. Since the results for the high resolution cases are almost identical, suggesting that this is a grid converged solution, for such cases we only include plots for $k=4$ and $k=7$, while all the low resolution cases are shown. It can be observed that all the considered combinations of polynomial degrees and space resolutions result in an accurate representation of the first two modes, while the third and the fourth modes are better represented when using fewer elements with higher polynomial degree, especially concerning the build-up of electrostatic energy for $t \in[20,40]$. To assess the role of the numerical dissipation, we plot in Figure 7.3 the $L^{2}$ norm of the distribution function. Here, it can be observed that the behaviour of the scheme is always dissipative, as anticipated in $\S 4.2$; the numerical dissipation is larger for lower polynomial degrees. The $L^{1}$ norm of the distribution function is reported in Figure 7.4. Since the number of particles is conserved up to machine precision by construction, changes in $\|f\|_{L^{1}}$ are due to violations of the positivity constraint $f(t, x) \geq 0$ implied by (2.11). Here, one can notice that negative values are produced roughly at the same rate for different values of $k$, and they are then dissipated at a rate which is inversely proportional to the polynomial degree; clearly, this is consistent with the numerical dissipation pattern resulting from Figure 7.3. For all the considered simulations, the total energy shows deviations within a range $\mathcal{O}\left(10^{-12}\right)$ : this is expected, since the total energy is conserved up to errors introduced by the time discretization, and the time-step is small.

The second test case considered in this section is the so-called "bump-on-tail" instability. This problem has been considered in [Sho79] and some additional reference results are available in [NY99, $\left.\mathrm{CMS}_{10}, \mathrm{MSM}^{+} 13\right]$. The computational domain is $\Omega_{\boldsymbol{x}}=(0,20 \pi)$ and $\Omega_{\boldsymbol{v}}=(-9,9)$ and the initial condition is

$$
f_{0}(x, v)=\frac{1}{10 \sqrt{2 \pi}}(1+A \cos (\kappa x))\left(9 \mathrm{e}^{-\frac{v^{2}}{2}}+\frac{1}{v_{\mathrm{th}}} \mathrm{e}^{-\frac{1}{2}\left(\frac{v-v_{d}}{v_{\mathrm{th}}}\right)^{2}}\right)
$$

with $A=0.04, \kappa=0.3, v_{d}=4.5$ and $v_{\text {th }}=0.5 . \quad \mathcal{T}_{\boldsymbol{x}}$ is composed of 18 uniform elements, while for $\mathcal{T}_{\boldsymbol{v}}$ we use 12 nonuniform elements with boundaries $[-9,-5.5,-3$, $-1,0,1,2,3,4,5,6,7.5,9]$. Notice that it is important that the number of elements in $\mathcal{T}_{\boldsymbol{x}}$ is a multiple of three to represent correctly the periodicity of the solution. The polynomial order is $k=6$. Figure 7.5 shows the distribution function at time $t=50$ using the four discretizations: CM, SCVU, UM, UECM. For the first three methods we set $c_{11}=h^{-1}(k+1)^{2}$, with $h=\frac{1}{2}\left(\left|K_{\boldsymbol{x}}\right|+\left|K_{\boldsymbol{v}}\right|\right)$, while for the energy conserving upwind method we set $c_{11}=0$. The time-step is $\Delta t=3.75 \cdot 10^{-3}$, so that the time discretization error is negligible. Clearly, the use of an upwind flux in the $x$ direction is required in order to obtain a solution free from spurious oscillations. The solution obtained with the centered method becomes unstable for larger times, while the solution computed with upwinding in the sole $v$ direction is stable and, 

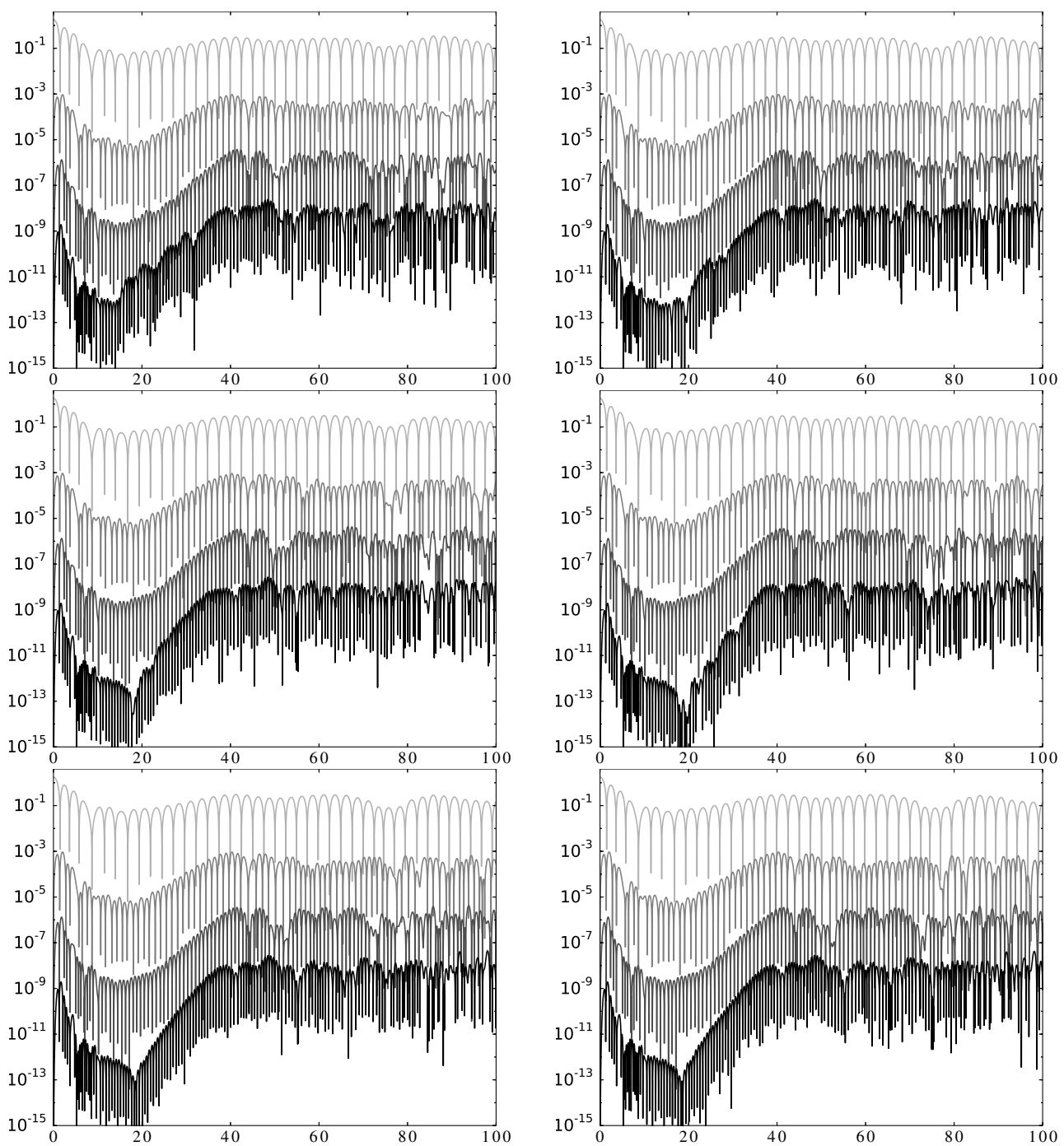

FIG. 7.2. Nonlinear Landau damping, time evolution of the electrostatic energy for the first four modes, $n$ increases from light gray to black. To separate the curves of the four modes, for each mode we plot $100^{-n+1} \sqrt{\mathscr{E}_{w, n}^{\mathrm{el}}}$. Results for the low resolution cases $k=4$ and $56 \times 112$ elements (top, left), $k=5$ and $47 \times 94$ elements (top, right), $k=6$ and $40 \times 80$ elements (center, left), $k=7$ and $35 \times 70$ (center, right), and for two high resolution cases $k=4$ and $112 \times 224$ elements (bottom, left) and $k=7$ and $70 \times 140$ elements (bottom, right).

for $t \geq 100$, the oscillations are mild and a qualitative agreement with the solutions obtained with the two fully upwind methods is observed. The solutions obtained with the two upwind methods are very similar during the whole time evolution. Figure 7.6 displays the $L^{2}$ and $L^{1}$ norms of the distribution function for the three stable methods. These plots confirm that the two solutions obtained with the fully upwind methods are very similar, and show that, despite the fact that the $L^{2}$ norm of the solution is dissipated roughly at the same rate, the violation of the positivity constraint $f(t, x) \geq$ 0 is significantly larger when the upwind method is used only in the velocity space. 

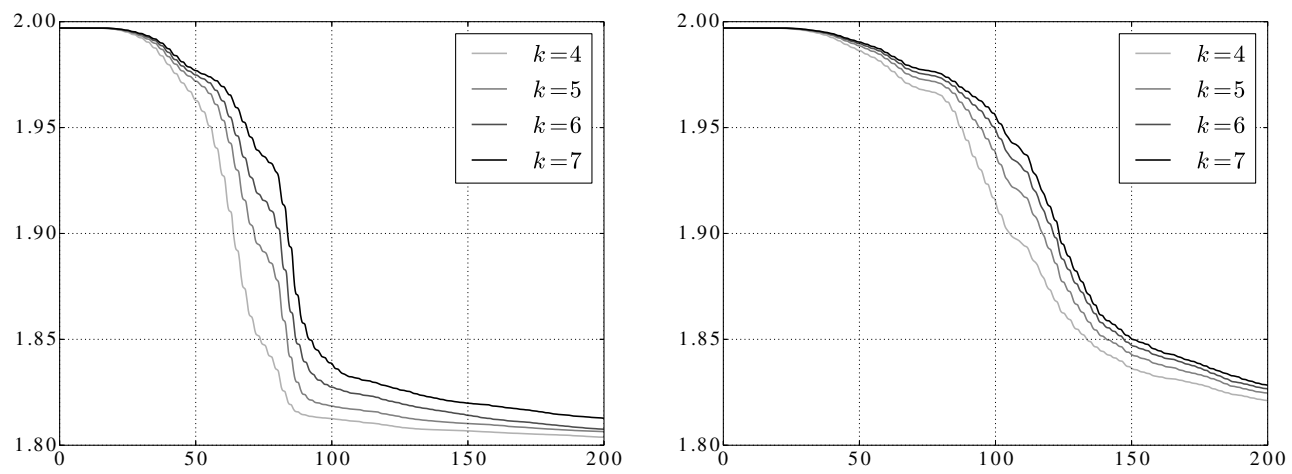

FIG. 7.3. Nonlinear Landau damping, time evolution of the $L^{2}$ norm of the distribution function for the low resolution runs (left) and the high resolution ones (right).
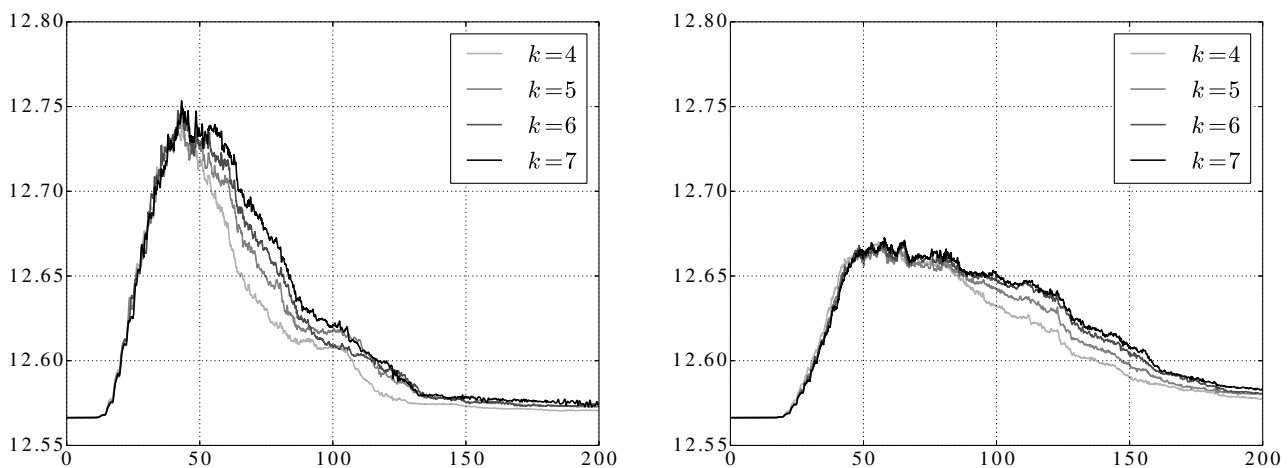

FIG. 7.4. Nonlinear Landau damping, time evolution of the $L^{1}$ norm of the distribution function for the low resolution runs (left) and the high resolution ones (right).

Concerning the total energy conservation, we plot the time evolution of the total energy in Figure 7.7 for different time-steps, the largest of which is the maximum time-step resulting in a stable computation. It can be seen that, while for the upwind method the deviation of the total energy is independent from $\Delta t$ (except for the very beginning of the computation) and is thus dominated by the space discretization error, for the energy conserving, upwind method the energy is exactly conserved in the limit $\Delta t \rightarrow 0$. Finally, concerning the momentum conservation, we mention that both upwind methods show variation of the total momentum within $0.1 \%$ of the initial condition, and these variations are almost independent from the time-step.

The third test case is the two-stream instability, for which we refer to [CMS10, HGMM12]. The initial condition is

$$
f_{0}(x, v)=\frac{1}{2 \sqrt{2 \pi} v_{\mathrm{th}}}(1+A \cos (\kappa x))\left(\mathrm{e}^{-\frac{1}{2}\left(\frac{v-v_{d}}{v_{\mathrm{th}}}\right)^{2}}+\mathrm{e}^{-\frac{1}{2}\left(\frac{v+v_{d}}{v_{\mathrm{th}}}\right)^{2}}\right)
$$

with $v_{\text {th }}=0.3, A=0.05, \kappa=1, v_{d}=0.99$. The computational domain is $\Omega_{x}=$ $(0,26 \pi), \Omega_{\boldsymbol{v}}=(-8,8)$, and we use a mesh composed of 52 elements in $x$ and 120 elements in $v$; the polynomial order is $k=5$ and the time-step $\Delta t=5 \cdot 10^{-3}$. There are many aspects of this test case which can be considered, and a thorough discussion can be found in [HGMM12]. Here, we are mostly interested in using this test as an example of a problem exhibiting very sharp gradients in the distribution function, 

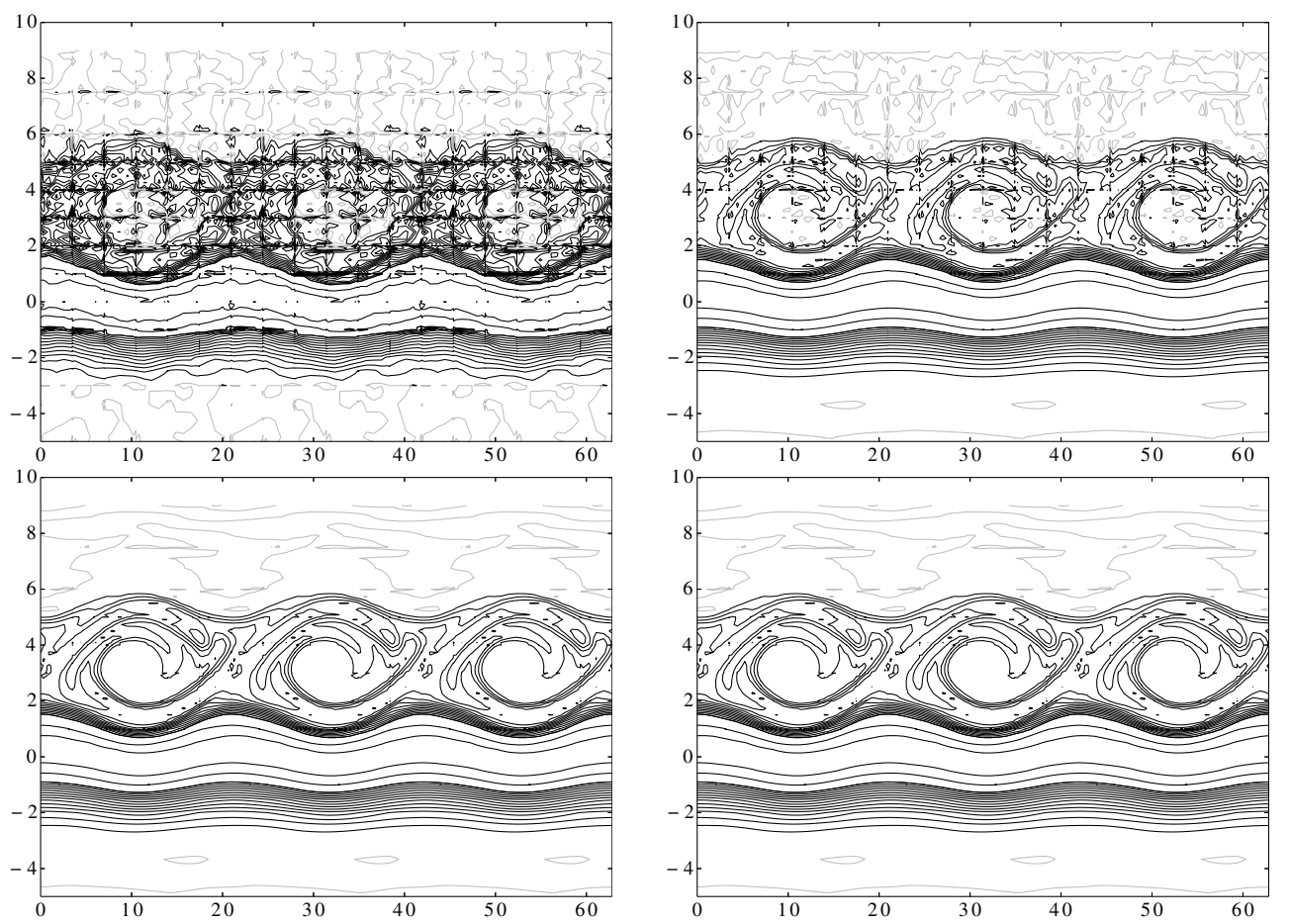

FIG. 7.5. Bump-on-tail test case, contour plot of the distribution function at $t=50$, zero contour in gray. Results for the CM (top, left), SCVU (top, right), UM (bottom, left), and UECM (bottom, right).
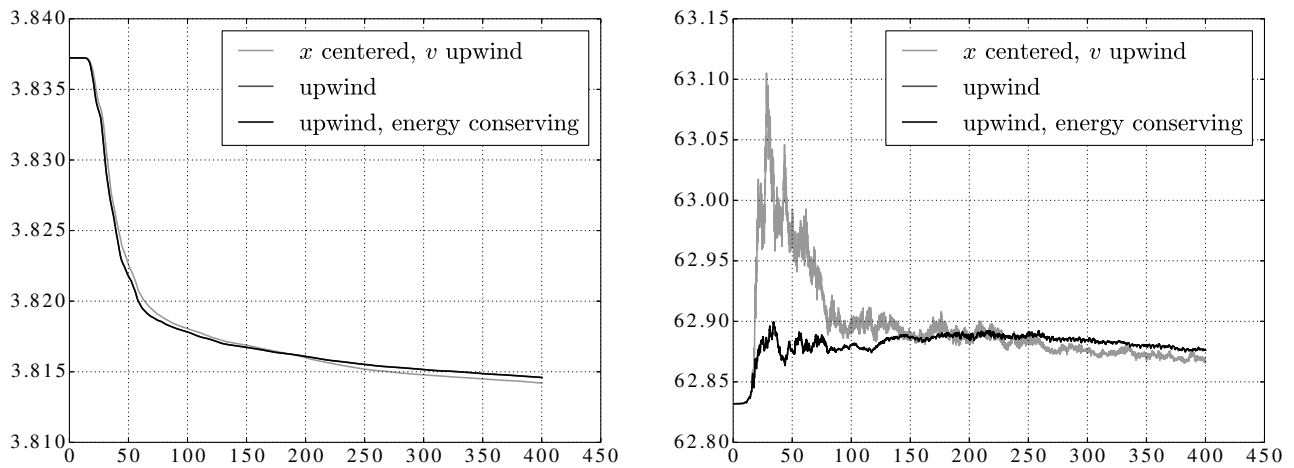

FIG. 7.6. Bump-on-tail test case, time evolution of the $L^{2}$ (left) and $L^{1}$ (right) norms of the distribution function. The two curves of the fully upwind methods are almost superimposed.

thus representing a challenging problem for the stability of the numerical method. For this reason, the resolution is rather coarse: each wave period in $x$ is resolved with four elements and the relevant dynamics in $v$ is resolved with $\approx 15$ elements. During the initial phase of the computation, the instability develops and thirteen electron hole-like structures are formed for $20 \leq t \leq 40$, which are then stable until $t \approx 110$. At this point, because of the truncation errors introduced by the numerical computation, the periodicity, as well as the symmetry, of the solution are destroyed and these structures start merging, resulting into fewer, larger structures. This is the 

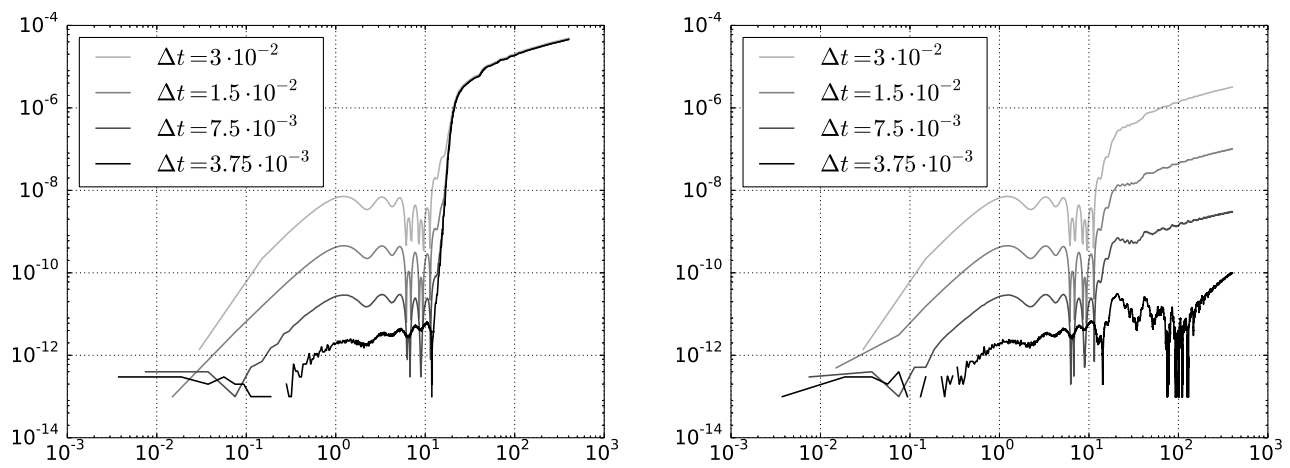

FIG. 7.7. Total energy deviation $\left|\mathscr{E}_{w}(t)-\mathscr{E}_{w}(0)\right|$ for the bump-on-tail test case for different time-steps; a logarithmic scale is used for the time axis to highlight both the initial transient and the long term behaviour. Results for the UM (left) and the UECM (right).

process that leads to the sharpest gradients in the distribution function. At the final time $t=1000$ only two electron hole-like structure are still present. The whole process can be seen in Figure 7.8 for the UECM computation (with $c_{11}=0$ ); similar results, not included here, are obtain with the UM (using $c_{11}=h^{-1}(k+1)^{2}$ as in the previous test case). Indeed, the solutions computed with UECM and UM are very similar, as it is also confirmed by Figure 7.9, where the $L^{2}$ and $L^{1}$ norms of the distribution function are displayed. Notice the "staircase" pattern in the $L^{2}$ norm of the solution: rapid changes in this norm correspond to strong numerical dissipation, which takes place during the formation of the electron hole-like structures for $t \approx 20$ and during the merging of such structures occurring, for UECM, at times 110, 350 and 570. The fact that the secondary merging happens at different times for the UM compared to the UECM should not be of concern, since this process is triggered by the truncation errors. The main differences between the UM and the UECM can be found in the total energy balance and in the presence of numerical overshoots and undershoots of the distribution function; these quantities are shown in Figure 7.10. During the formation of the electron hole-like structures at $t \approx 20$ the UM shows a significant change in the total energy, and both methods display over- and undershoots of the same entity. For larger times, during the merging of these structures, the UM shows a second, more pronounced change in the total energy and both methods exhibit severe over- and undershoots, with the UECM being characterized by overshoots twice as large as the UM. The larger overshoots of the UECM method are due to few, isolated points located on the $v=0$ axis at positions where $\tilde{\boldsymbol{E}}_{h}$ has large jumps going from $v<0$ to $v>0$; for this reason, one could regard these larger overshoots as the "price" to be paid for the introduction of an energy conserving reconstruction of the electric field. We mention that it might be possible to cure this effect with the introduction of some smoothing mechanism for elements close to the $v=0$ axis, or by introducing a smooth transition in the definition of the upwind scheme exploiting the upwinding parameter $\omega$ in (3.7), but we do not explore this topic any further in this work. Finally, we mention that the total momentum remains with $\mathcal{O}\left(10^{-12}\right)$ for $t \leq 110$; after this point the symmetry of the solution is lost and fluctuations in the total momentum appear which are of the order $\mathcal{O}\left(10^{-3}\right)$ for both methods. 

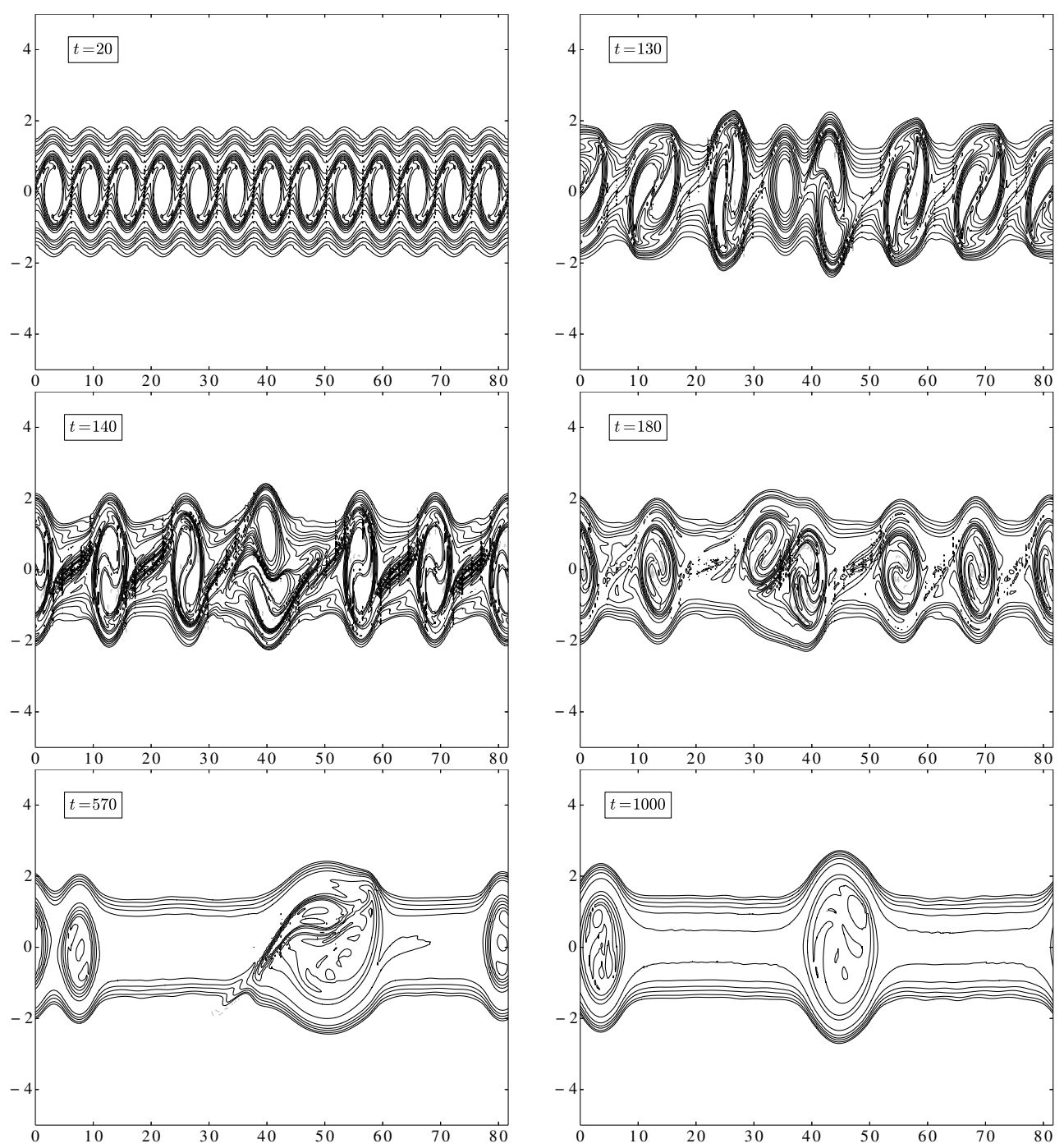

FIG. 7.8. Two-stream instability test case: contour plots of the distribution function obtained with the UECM at different time levels.

7.3. Two-dimensional test cases. In this section, we consider a two dimensional version of the two-stream instability problem. The initial condition is

$$
\begin{aligned}
f_{0}\left(x^{1}, x^{2}, v^{1}, v^{2}\right)= & \frac{1}{4 \pi v_{\mathrm{th}}^{1} v_{\mathrm{th}}^{2}}\left(1-A^{1} \sin \left(\kappa^{1} x^{1}\right)-A^{2} \sin \left(\kappa^{2} x^{2}\right)\right) \\
& \left(\mathrm{e}^{-\frac{1}{2}\left[\left(\frac{v^{1}-v_{d}}{v_{\mathrm{th}}^{1}}\right)^{2}+\left(\frac{v^{2}}{v_{\mathrm{th}}^{2}}\right)^{2}\right]}+\mathrm{e}^{\left.-\frac{1}{2}\left[\left(\frac{v^{1}+v_{d}}{v_{\mathrm{th}}^{1}}\right)^{2}+\left(\frac{v^{2}}{v_{\mathrm{th}}^{2}}\right)^{2}\right]\right)},\right.
\end{aligned}
$$

with $v_{\text {th }}^{1}=1.0, v_{\text {th }}^{2}=0.5, A^{1}=0.05, A^{2}=0.25, \kappa^{1}=\kappa^{2}=0.2$, and $v_{d}=2.4$. The perturbation in $x^{1}$ direction is small since its purpose is triggering the instability of the two streams, which are directed along $x^{1}$. In the $x^{2}$ direction the velocity has a Gaussian distribution, so we apply a relatively large perturbation to obtain a Landau 

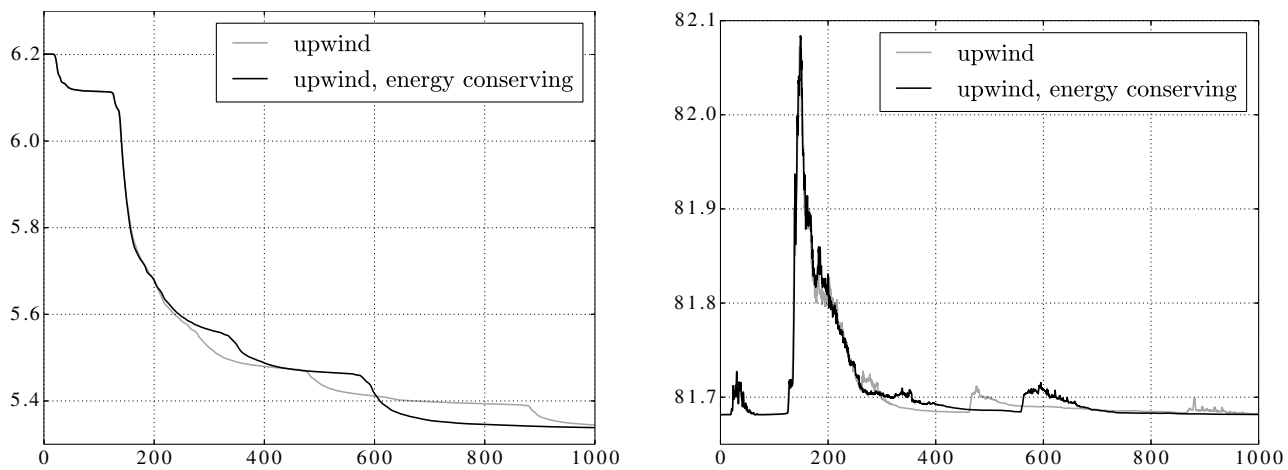

FIG. 7.9. Two-stream instability test case: time evolution of the $L^{2}$ norm (left) and $L^{1}$ norm (right) of the distribution function computed with the UM and the UECM.
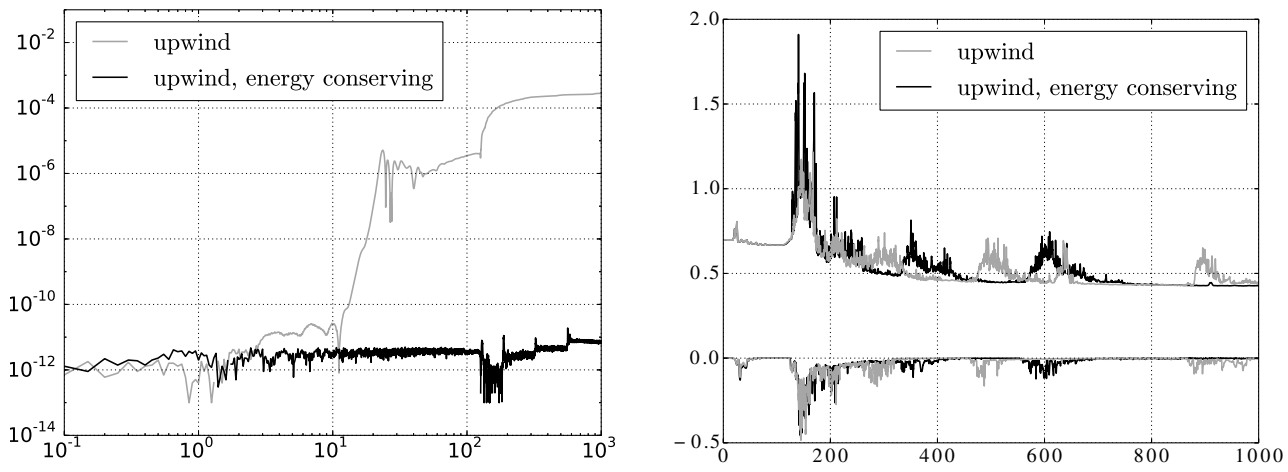

FIG. 7.10. Two-stream instability test case: total energy deviation $\left|\mathscr{E}_{w}(t)-\mathscr{E}_{w}(0)\right|$ (left, a logarithmic scale is used also for the time axis) and time evolution of $\max \left(f_{h}\right)$ and $\min \left(f_{h}\right)$ (right) for the UP and UECM.

damping behaviour. The chosen combination $\kappa^{2}, v_{\text {th }}^{2}$ produces a very small damping, in order to ensure that the problem maintains its two-dimensional character. The computational domain is $\Omega_{\boldsymbol{x}}=(0,10 \pi)^{2}, \Omega_{\boldsymbol{v}}=(-10,10)^{2}$, and the mesh is composed of $5 \times 5$ uniform elements in $\Omega_{\boldsymbol{x}}$ and $64 \times 32$ uniform elements in $\Omega_{\boldsymbol{v}}$ with $k=5$. The computations are performed with the UECM setting $c_{11}=h^{-1}(k+1)^{2}$. The time evolution of the electrostatic energy and of the $L^{2}$ norm of the distribution function are plotted in Figure 7.11, where it can be seen that all the quantities show the expected behaviour. Figure 7.12 shows two phase-space sections for $x^{2}=0, v^{2}=0$ of the distribution function for time levels $t=12$ and $t=100$. Here, it can be seen that the growth of the instability in the two streams has lead to a pattern similar to Figure 7.8. For this computation, energy is conserved within relative fluctuations of order $\mathcal{O}\left(10^{-10}\right)$, while momentum exhibits fluctuations of order $\mathcal{O}\left(10^{-2}\right)$.

7.4. Accuracy and efficiency of the time discretization. In this section, we compare the RK4 and ERB2 time integration methods discussed in $\S 6$. To this end, we consider the "bump-on-tail" instability test case with the same setup described in $\S 7.2$. A reference solution is obtained using the RK4 method with $\Delta t=3.75 \cdot 10^{-3}$, which is eight times smaller than the maximum stable time-step for RK4, namely $\Delta t^{R K 4}=3.0 \cdot 10^{-2}$. We now solve this problem for $t \in(0,61.44)$ with the ERB2 

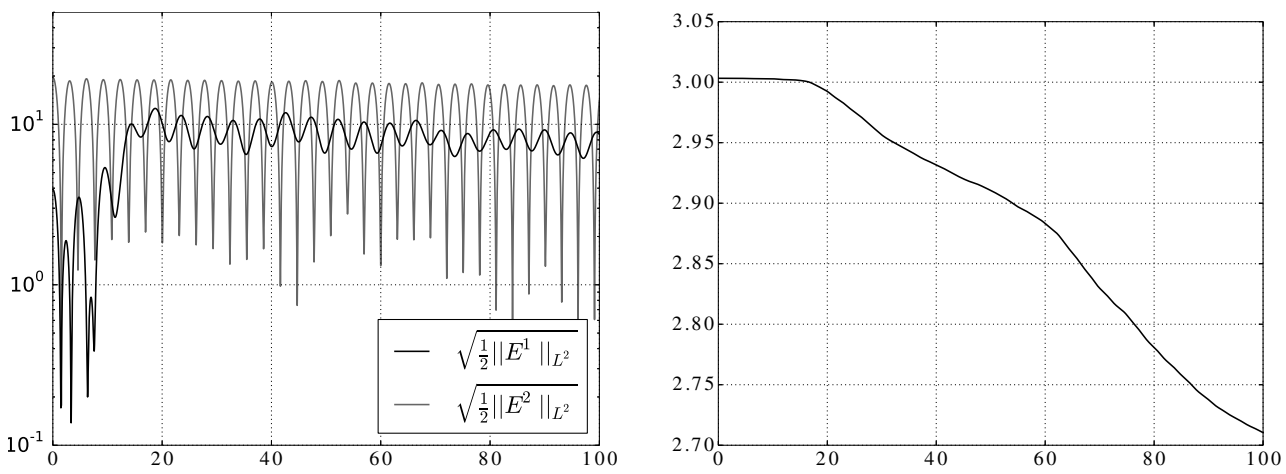

FIG. 7.11. Two-dimensional two-stream instability: time evolution of the electrostatic energy, separating the contributions of the electric field in the $x^{1}$ and $x^{2}$ directions (left) and time evolution of $\left\|f_{h}\right\|_{L^{2}}$ (right).
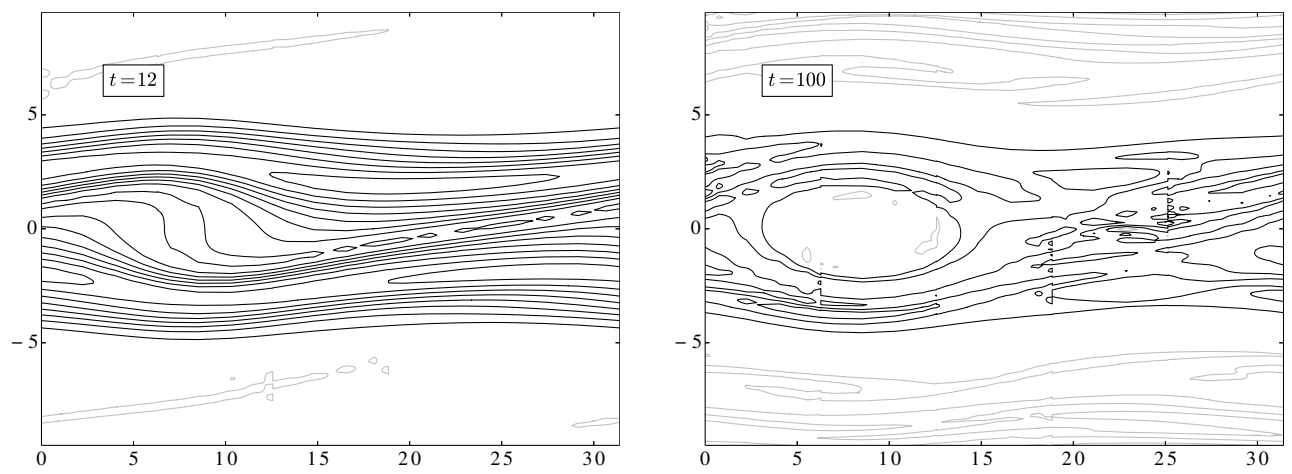

FIG. 7.12. Two-dimensional two-stream instability: contour plot of the distribution function on the plane $x^{2}=0, v^{2}=0$ for time levels $t=12$ and $t=100$. The zero contour line is shown in gray.

method using both Krylov iterations and Leja point interpolation for the computation of the matrix exponential, for different time-steps and different tolerances $\varepsilon_{\text {Kry }}$ and $\varepsilon_{\mathrm{Lej}}$, and compute the $L^{2}$ error norm at $t=61.44$ and the efficiency index $\eta_{\text {eff }}$, defined in (6.6), resulting from the average number of iterations per time step. As discussed in $\S 6$, such an index quantifies the ratio of the computational costs of the RK integrator and of the exponential one. The interval for the interpolation with the Leja points is chosen as [ $-76 \Delta t, 0]$, based on an estimate of the eigenvalues of $\mathcal{J}_{\mathbf{R}}^{n}$. The results are collected in Table 7.2 for the Krylov iteration method and in Table 7.3 for the Leja point interpolation one. For comparisons, the RK4 method with timestep $\Delta t^{\mathrm{RK} 4}$ yields an error equal to $9.5 \cdot 10^{-5}$. Clearly, for $\Delta t^{\mathrm{ERB} 2} \leq \Delta t^{\mathrm{RK} 4}$, ERB2 is not competitive with RK4, since it tends to be both less accurate and less efficient. However, for $\Delta t^{\mathrm{ERB} 2}>\Delta t^{\mathrm{RK} 4}$, ERB2 can be up to twice more efficient than RK while the resulting errors are still of the order $\mathcal{O}\left(10^{-2}\right)$, thereby allowing a trade-off between accuracy and efficiency which is not possible with RK4. Comparing now the two options for the computation of the exponential matrix, despite the fact that for each combination of accuracy and time-step one option can perform better than the other, no systematic pattern emerges, so that none of them seems to be clearly superior for the considered problem. On the one hand, for small time-steps the $L^{2}$ error in the solution is dominated for both methods by the error in the computation 
TABLE 7.2

$L^{2}$ error norms and efficiency index (6.6) for the ERB2 method with Krylov iterations, for varying accuracy in the computation of the exponential matrix; the problem considered here is the "bump-on-tail" instability, using the upwind, energy conserving method with the same setup of $§ 7.2$.

\begin{tabular}{|c||c|c||c|c||c|c||}
\hline \multicolumn{1}{|c||}{} & \multicolumn{2}{c||}{$\varepsilon_{\text {Kry }}=10^{-9}$} & \multicolumn{2}{c||}{$\varepsilon_{\text {Kry }}=10^{-6}$} & \multicolumn{2}{c||}{$\varepsilon_{\text {Kry }}=10^{-3}$} \\
$\Delta t^{\mathrm{ERB} 2}$ & $\left\|f_{\text {ref }}-f_{h}\right\|_{L^{2}}$ & $\eta_{\text {eff }}$ & $\left\|f_{\text {ref }}-f_{h}\right\|_{L^{2}}$ & $\eta_{\text {eff }}$ & $\left\|f_{\text {ref }}-f_{h}\right\|_{L^{2}}$ & $\eta_{\text {eff }}$ \\
\hline $7.5 \cdot 10^{-3}$ & $4.6 \cdot 10^{-5}$ & 0.17 & $4.2 \cdot 10^{-4}$ & 0.26 & $6.3 \cdot 10^{-1}$ & 0.39 \\
\hline $1.5 \cdot 10^{-2}$ & $1.8 \cdot 10^{-4}$ & 0.30 & $1.9 \cdot 10^{-4}$ & 0.42 & $1.2 \cdot 10^{-1}$ & 0.70 \\
\hline $3.0 \cdot 10^{-2}$ & $7.4 \cdot 10^{-4}$ & 0.47 & $7.5 \cdot 10^{-4}$ & 0.71 & $2.4 \cdot 10^{-1}$ & 1.24 \\
\hline $6.0 \cdot 10^{-2}$ & $3.0 \cdot 10^{-3}$ & 0.69 & $3.0 \cdot 10^{-3}$ & 1.00 & $6.1 \cdot 10^{-2}$ & 1.53 \\
\hline $1.2 \cdot 10^{-1}$ & $1.3 \cdot 10^{-2}$ & 0.91 & $1.3 \cdot 10^{-2}$ & 1.25 & $3.1 \cdot 10^{-2}$ & 1.76 \\
\hline $2.4 \cdot 10^{-1}$ & $7.9 \cdot 10^{-2}$ & 1.14 & $7.9 \cdot 10^{-2}$ & 1.46 & $8.5 \cdot 10^{-2}$ & 1.89 \\
\hline $4.8 \cdot 10^{-1}$ & $8.8 \cdot 10^{-1}$ & 1.35 & $8.8 \cdot 10^{-1}$ & 1.62 & $8.9 \cdot 10^{-1}$ & 1.99 \\
\hline
\end{tabular}

TABLE 7.3

$L^{2}$ error norms and efficiency index (6.6) for the ERB2 method with Leja point interpolation, for varying accuracy in the computation of the exponential matrix; the problem considered here is the "bump-on-tail" instability, using the upwind, energy conserving method with the same setup of $\S 7.2$.

\begin{tabular}{|c||c|c||c|c||c|c||}
\hline \multicolumn{1}{|c||}{} & \multicolumn{2}{c||}{$\varepsilon_{\text {Lej }}=10^{-3}$} & \multicolumn{2}{c||}{$\varepsilon_{\text {Lej }}=10^{-2}$} & \multicolumn{2}{c||}{$\varepsilon_{\text {Lej }}=10^{-1}$} \\
$\Delta t^{\mathrm{ERB} 2}$ & $\left\|f_{\text {ref }}-f_{h}\right\|_{L^{2}}$ & $\eta_{\text {eff }}$ & $\left\|f_{\text {ref }}-f_{h}\right\|_{L^{2}}$ & $\eta_{\text {eff }}$ & $\left\|f_{\text {ref }}-f_{h}\right\|_{L^{2}}$ & $\eta_{\text {eff }}$ \\
\hline $7.5 \cdot 10^{-3}$ & $4.6 \cdot 10^{-5}$ & 0.15 & $4.6 \cdot 10^{-5}$ & 0.17 & $5.3 \cdot 10^{-5}$ & 0.20 \\
\hline $1.5 \cdot 10^{-2}$ & $1.8 \cdot 10^{-4}$ & 0.26 & $1.9 \cdot 10^{-4}$ & 0.32 & $5.9 \cdot 10^{-4}$ & 0.40 \\
\hline $3.0 \cdot 10^{-2}$ & $7.4 \cdot 10^{-4}$ & 0.49 & $7.7 \cdot 10^{-4}$ & 0.58 & $1.2 \cdot 10^{-2}$ & 0.80 \\
\hline $6.0 \cdot 10^{-2}$ & $3.0 \cdot 10^{-3}$ & 0.81 & $3.5 \cdot 10^{-3}$ & 1.02 & $2.3 \cdot 10^{-1}$ & 1.60 \\
\hline $1.2 \cdot 10^{-1}$ & $1.3 \cdot 10^{-2}$ & 1.24 & $1.2 \cdot 10^{-2}$ & 1.64 & $9.5 \cdot 10^{-2}$ & 1.95 \\
\hline $2.4 \cdot 10^{-1}$ & $7.9 \cdot 10^{-2}$ & 1.57 & $7.9 \cdot 10^{-2}$ & 1.58 & $1.0 \cdot 10^{-1}$ & 1.60 \\
\hline $4.8 \cdot 10^{-1}$ & $8.8 \cdot 10^{-1}$ & 1.44 & $8.8 \cdot 10^{-1}$ & 1.45 & 1.2 & 1.47 \\
\hline
\end{tabular}

of $\phi\left(\Delta t \mathcal{J}_{\mathbf{R}}^{n}\right)$, so that small values of $\varepsilon_{\text {Kry }}$ and $\varepsilon_{\text {Lej }}$ result in higher accuracy, and for the smallest considered tolerance the second order convergence rate of the ERB2 scheme can be observed. For larger time-steps, on the other hand, the limiting factor is the accuracy of the ERB2 scheme and no significant difference is observed reducing $\varepsilon_{\text {Kry }}$ or $\varepsilon_{\text {Lej }}$. The largest considered time step $\Delta t=0.48$ results in rather inaccurate solutions which, albeit being stable, are of little practical interest.

8. Conclusion and future developments. In this work, a spectral element DG formulation for the Vlasov-Poisson equation has been discussed, with particular attention to its discrete conservation properties. The scheme conserves naturally the number of particle, and a suitable reconstruction of the electric field has been discussed resulting in exact energy conservation (up to errors introduced by the time discretization). The accuracy and stability of the resulting method have been investigated using some classical test cases.

Concerning the time discretization of the problem, two methods have been considered: a classical RK scheme and a second order exponential time integrator. This latter method can be competitive in terms of computational efficiency when a moderate accuracy in the distribution function can be accepted.

The most natural continuation of this work concerns the use of higher order exponential integrators, which could allow large time steps without sacrificing the accuracy of the numerical solution.

Other possibilities that we plan to explore are the use of limiters for the control of the spurious oscillations, for instance along the lines of [ZS10], and the use of adaptive mesh refinement. 
Acknowledgments. Many ideas addressed in this work are the result of various discussions with Blanca Ayuso de Dios and Soheil Hajian.

We would also like to thank Luca Bonaventura, Daniel Y. Le Roux, Omar Maj, Michael Kraus and Philip J. Morrison for many interesting comments and suggestions.

The first author (Éric Madaule) worked at this paper during his Master Degree at the ENSEIRB-Matmeca engineering school in Bordeaux.

Appendix A. Dispersion analysis of the DG method. The target of the dispersion analysis is to assess to which extent the linear behaviour of the discretized system resembles that of the continuous one. While doing this, one also obtains some information about the spectral properties of the Jacobian matrix $\mathcal{J}_{\mathbf{R}}^{n}$ in (6.3), which significantly affect the stability of the time discretization for both RK4 and ERB4 and, for ERB2, also the convergence of the iterative computation of $\phi\left(\Delta t \mathcal{J}_{\mathbf{R}}^{n}\right)$, as discussed for instance in in [Saa91, HLS98, CVB04]. The dispersion analysis for DG schemes presents some additional difficulties compared to the standard one for finite difference methods due to the fact that, even on a uniform grid, not all the degrees of freedom are equivalent; this is the motivation for including the present section.

Rather than analyzing the complete Vlasov-Poisson problem, we focus here on the one-dimensional advection equation, which, albeit being much simpler, still represents an important feature of the model. Moreover, we only consider linear elements; much more general results can be found in [LC05, Le 13].

Let us thus consider the problem

$$
\partial_{t} u+a \partial_{x} u=0
$$

for constant $a>0$. Introducing a uniform grid in space with elements of size $h$ and discretizing (A.1) with piecewise linear polynomials and upwind numerical fluxes, one arrives at the ODE system

$$
\begin{aligned}
\frac{h}{3} \dot{u}_{i}^{L}+\frac{h}{6} \dot{u}_{i}^{R}+\frac{a}{2}\left(u_{i}^{L}+u_{i}^{R}\right)-a u_{i-1}^{R} & =0 \\
\frac{h}{6} \dot{u}_{i}^{L}+\frac{h}{3} \dot{u}_{i}^{R}-\frac{a}{2}\left(u_{i}^{L}+u_{i}^{R}\right)+a u_{i}^{R} & =0,
\end{aligned}
$$

where $u_{i}^{L}$ and $u_{i}^{R}$ are the degrees of freedom collocated at the leftmost and rightmost point of the $i$-th element, respectively. Such an equation can be written in matrix notation as

$$
\mathcal{M} \dot{\mathbf{u}}+\mathcal{A} \mathbf{u}=0 .
$$

In the dispersion analysis, we look for solutions of $(\mathrm{A} .3)$ of the form $\mathbf{u}(t)=\hat{\mathbf{u}} \mathrm{e}^{I \omega t}$, where $\hat{\mathbf{u}}$ is an arbitrary but fixed vector, $\omega \in \mathbb{C}$ and $I=\sqrt{-1}$. Notice that, once such a solution has been found, it satisfies

$$
I \omega \mathcal{M} \hat{\mathbf{u}}+\mathcal{A} \hat{\mathbf{u}}=0
$$

and, observing that (A.3) can be recast in form (6.3) upon multiplying by $\mathcal{M}^{-1}$ and setting $\mathcal{J}_{\mathbf{R}}=-\mathcal{M}^{-1} \mathcal{A}$, we see that $\lambda=I \omega$ is an eigenvalue of $\mathcal{J}_{\mathbf{R}}$ and $\hat{\mathbf{u}}$ is the corresponding eigenvector; this provides the link with the study of the spectral properties of $\mathcal{J}_{\mathbf{R}}$. As anticipated, the difficulty in performing the dispersion analysis of (A.3) is the presence of two kinds of degrees of freedom, the "L" values and the "R" values, which are not equivalent because they solve two different equations, namely $(\mathrm{A} .2)_{1}$ and $(\mathrm{A} .2)_{2}$. We proceed by making the following ansatz:

$$
\left[\begin{array}{c}
u_{i}^{L}(t) \\
u_{i}^{R}(t)
\end{array}\right]=\left[\begin{array}{l}
\hat{u}^{L} \\
\hat{u}^{R}
\end{array}\right] \mathrm{e}^{I(\omega t-\kappa h i)},
$$


where $\hat{u}^{L}$ and $\hat{u}^{R}$ are two scalar constants which do not depend on $t$ or $i$. Substituting (A.4) into (A.3) we arrive at the condition

$$
\left[\begin{array}{cc}
2 I \tilde{\omega}+3 & I \tilde{\omega}+3(1-2 \Psi) \\
I \tilde{\omega}-3 & 2 I \tilde{\omega}+3
\end{array}\right]\left[\begin{array}{c}
\hat{u}^{L} \\
\hat{u}^{R}
\end{array}\right]=0
$$

where $\tilde{\omega}=\frac{h \omega}{a}$ and $\Psi=\mathrm{e}^{I \kappa h}$. Equation (A.5) is a $2 \times 2$ generalized eigenvalue problem, providing the dispersion relation of the discrete system. To complete the dispersion analysis, the modes of the discrete system must be compared with those of the continuous one (A.1), which are easily seen to be

$$
\mathrm{e}^{I(\omega t-\kappa x)}, \quad \omega=a \kappa .
$$

The difficulty now arises because, while (A.6) provides one frequency $\omega$ for each wave number $\kappa$, equation (A.5) admits two solutions for each $\kappa$. To deal with this aspect, we need to take into account that the space variation, and thus the wave number, of a discrete mode depends on both $\kappa$ and the two values $\hat{u}^{L}, \hat{u}^{R}$. This can be done as follows. For a generic value $\kappa \in\left[-\frac{\pi}{h}, \frac{\pi}{h}\right]$, let us indicate by $\omega_{\kappa, j}$ the two solutions of (A.5) and by $\hat{u}_{\kappa, j}^{L}, \hat{u}_{\kappa, j}^{R}$ the components of the corresponding eigenvectors, for $j=1,2$. We can introduce $\varrho_{\kappa, j}>0$ and $\vartheta_{\kappa, j} \in[-\pi, \pi)$ such that

$$
\hat{u}_{\kappa, j}^{R}=\varrho_{\kappa, j} \mathrm{e}^{-I \vartheta_{\kappa, j}} \hat{u}_{\kappa, j}^{L},
$$

so that from (A.4) the corresponding mode is (up to a multiplicative constant)

$$
u_{i}^{L}(t ; \kappa, j)=\mathrm{e}^{I\left(\omega_{\kappa, j} t-\kappa h i\right)}, \quad u_{i}^{R}(t ; \kappa, j)=\varrho_{\kappa, j} \mathrm{e}^{I\left(\omega_{\kappa, j} t-\kappa h i-\vartheta_{\kappa, j}\right)} .
$$

The "L" component of (A.7) is consistent with the sampling at $x_{i}=h i$ of the continuous mode

$$
\mathrm{e}^{I\left(\omega_{\kappa, j} t-\left(\kappa+\frac{2 \pi}{h} p\right) x\right)}
$$

for an arbitrary integer $p$. We now choose $p$ in order to maximize the agreement with the "R" component of (A.7), thereby completely identifying the continuous mode which should be compared with the discrete one. To this end, we define $p$ according to the following condition: minimize $\left|\kappa+\frac{2 \pi}{h} p\right|$ among the values $p$ such that

$$
|\kappa h+2 \pi p| \geq\left|\vartheta_{\kappa, j}\right|, \quad \operatorname{sign}(\kappa h+2 \pi p)=\operatorname{sign}\left(\vartheta_{\kappa, j}\right) .
$$

An example of this procedure is shown in Figure A.1. Here, we fix $\kappa h=1.885$ in (A.5), obtaining $\tilde{\omega}_{1}=1.930+0.142 I, \tilde{\omega}_{2}=-3.832+3.240 I$ and $\vartheta_{1}=1.508, \vartheta_{2}=-2.548$; according to (A.9), these values yield $p=0$ and $p=-1$, respectively, and the resulting discrete and continuous modes are shown. The dispersion relation $\operatorname{Re}(\omega(\kappa))$ is shown in Figure A.2, which is similar to the dispersion equation obtained for standard second order finite differences formulations; the same figure also shows the complete set of eigenvalues $\lambda$ of $\mathcal{J}_{\mathbf{R}}=-\mathcal{M}^{-1} \mathcal{A}$ according to (A.3).

To provide an indication concerning the relevance of this analysis for the complete Vlasov-Poisson system, we plot in Figure A.3 the Ritz values of $\mathcal{J}_{\mathbf{R}}^{n}$ for the "bump-ontail" instability problem with the same setup used in $\S$ 7.2. As explained in [SSS09], these values are easily obtained as a byproduct of the computation of $\phi\left(\Delta t \mathcal{J}_{\mathbf{R}}^{n}\right)$ and are approximations of the eigenvalues of $\mathcal{J}_{\mathbf{R}}^{n}$. Notice the similarity in the pattern with Figure A.2, right; similar results can be also observed in [DJ04], Figure 3. 

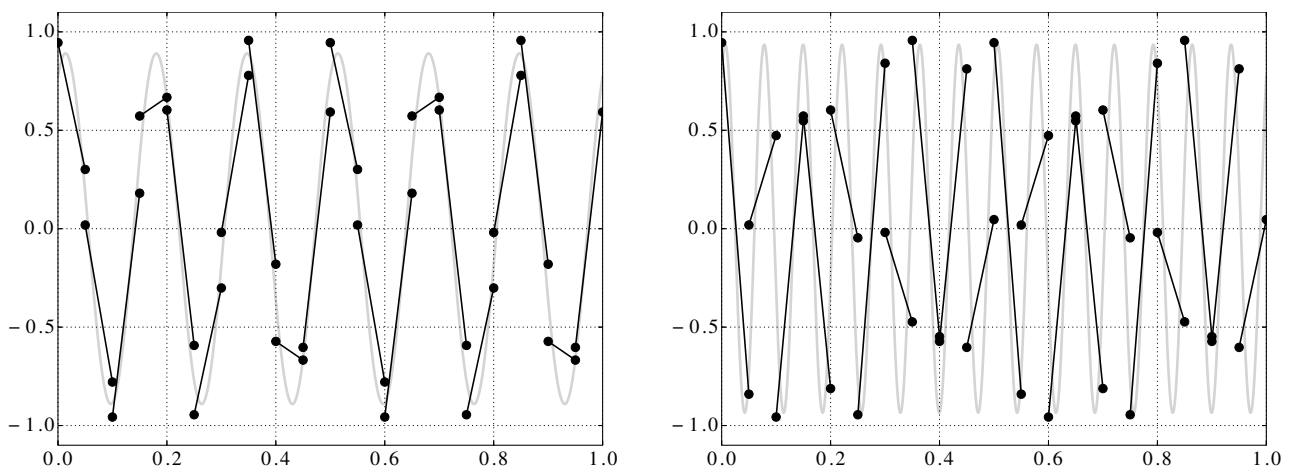

FIG. A.1. DG discretization of the advection equation with linear elements, plots of the two modes associated with $\kappa h=1.885$; discrete modes are represented in black while the corresponding continuous ones in gray. Results for $\tilde{\omega}_{1}=1.930+0.142 I$ (left) and $\tilde{\omega}_{2}=-3.832+3.240 I$ (right).
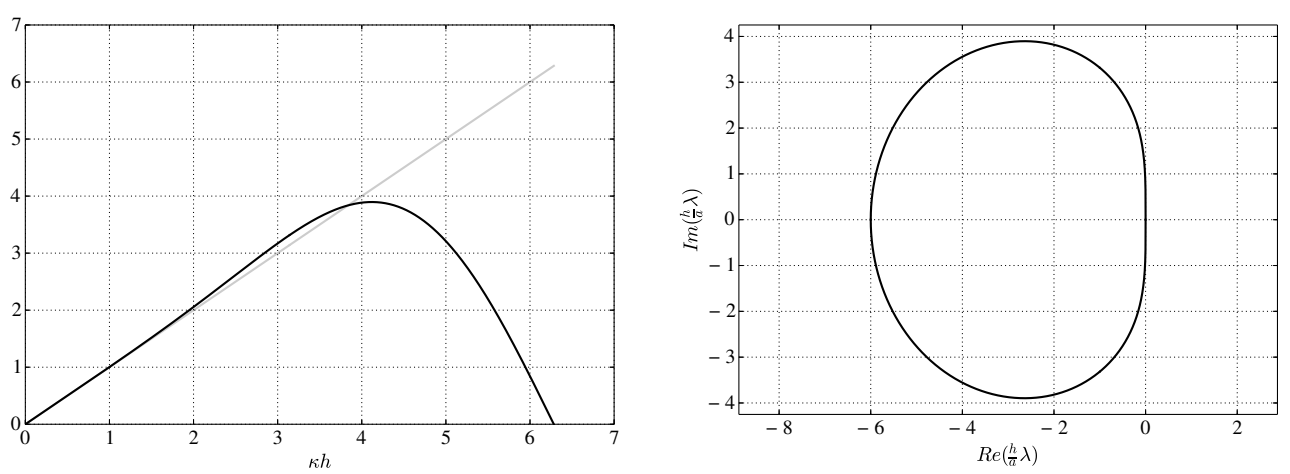

FIG. A.2. $D G$ discretization of the advection equation with linear elements: dispersion relation $\operatorname{Re}(\omega(\kappa))$, left, and values $\frac{h}{a} \lambda$ for $\lambda$ eigenvalue of $\mathcal{J}_{\mathbf{R}}=-\mathcal{M}^{-1} \mathcal{A}$ as resulting from (A.3), right.

\section{REFERENCES}

[AB85] Douglas N. Arnold and Franco Brezzi. Mixed and nonconforming finite-element methods - implementation, postprocessing and error-estimates. Rairo-Mathematical Modelling and Numerical Analysis - Modelisation Mathematique et Analyse Numerique, 19(1):7-32, 1985.

[ABCM02] Douglas N. Arnold, Franco Brezzi, Bernardo Cockburn, and L. Donatella Marini. Unified analysis of discontinuous Galerkin methods for elliptic problems. Siam Journal On Numerical Analysis, 39(5):1749-1779, January 2002.

[ACS11] Blanca Ayuso de Dios, José A. Carrillo, and Chi-Wang Shu. Discontinuous Galerkin methods for the one-dimensional Vlasov-Poisson system. Kinetic and Related Models, 4(4):955-989, December 2011.

[ACS12] Blanca Ayuso de Dios, José A. Carrillo, and Chi-Wang Shu. Discontinuous Galerkin methods for the multi-dimensional Vlasov-Poisson problem. Math. Models Methods Appl. Sci., 22(12):1250042-, June 2012.

[AS12] Blanca Ayuso de Dios and Hajian Soheil. High order and energy preserving discontinuous Galerkin methods for the Vlasov-Poisson system. Technical report, arXiv:1209.4025, 2012.

[Boy01] John P. Boyd. Chebyshev and Fourier Spectral Methods. Courier Dover Publications, 2001.

[BSC11] Sébastien Blaise and Amik St-Cyr. A dynamic $h p$-adaptive discontinuous Galerkin method for shallow-water flows on the sphere with application to a global tsunami simulation. Mon. Wea. Rev., 140(3):978-996, September 2011. 


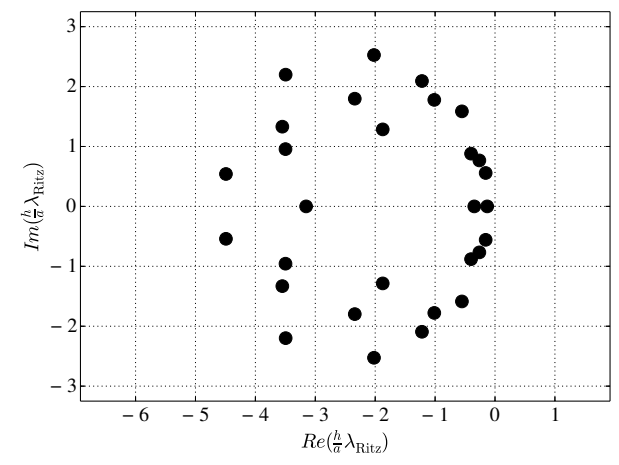

FIG. A.3. Ritz values for $\mathcal{J}_{\mathbf{R}}^{n}$ for the "bump-on-tail" test case. The values are scaled by $\frac{h}{a}$ with $h=\frac{20 \pi}{18 \cdot 6}, a=9$, representing an equivalent element size and the maximum velocity, to make them comparable with Figure A.2, left.

[CCPS01] Paul Castillo, Bernardo Cockburn, Ilaria Perugia, and Dominik Schötzau. An a priori error analysis of the local discontinuous Galerkin method for elliptic problems. SIAM Journal on Numerical Analysis, 38(5):1676-1706, January 2001.

[CCZ14a] Yingda Cheng, Andrew J. Christlieb, and Xinghui Zhong. Energy-conserving discontinuous Galerkin methods for the Vlasov-Ampère system. Journal of Computational Physics, 256(0):630-655, January 2014.

[CCZ14b] Yingda Cheng, Andrew J. Christlieb, and Xinghui Zhong. Energy-conserving discontinuous Galerkin methods for the Vlasov-Maxwell system. Journal of Computational Physics, 279(0):145-173, 2014.

[CGL09] Bernardo Cockburn, Jayadeep Gopalakrishnan, and Raytcho Lazarov. Unified hybridization of discontinuous Galerkin, mixed, and continuous Galerkin methods for second order elliptic problems. Siam Journal On Numerical Analysis, 47(2):1319-1365, 2009.

[CGM13] Yingda D. Cheng, Irene M. Gamba, and Philip J. Morrison. Study of conservation and recurrence of Runge-Kutta discontinuous Galerkin schemes for Vlasov-Poisson systems. Journal of Scientific Computing, 56(2):319-349, August 2013.

[CGMQ14] Andrew Christlieb, Wei Guo, Maureen Morton, and Jing-Mei Qiu. A high order time splitting method based on integral deferred correction for semi-Lagrangian Vlasov simulations. Journal of Computational Physics, 267(0):7-27, June 2014.

[CHQZ06] Claudio Canuto, Yousuff M. Hussaini, Alfio Quarteroni, and Thomas Zang. Spectral methods. Fundamentals in single domains. Springer Verlag, Berlin, 2006.

[CHQZ07] Claudio Canuto, Yousuff M. Hussaini, Alfio Quarteroni, and Thomas Zang. Spectral methods. Evolution to complex geometries and applications to fluid dynamics. Springer Verlag, Berlin, 2007.

[CHS90] Bernardo Cockburn, Suchung Hou, and Chi-Wang Shu. The Runge-Kutta local projection discontinuous Galerkin finite element method for conservation laws IV. The multidimensional case. Mathematics of Computation, 54:545-581, 1990.

[CK76] C.Z. Cheng and Georg Knorr. The integration of the Vlasov equation in configuration space. Journal of Computational Physics, 22(3):330-351, November 1976.

[CLS89] Bernardo Cockburn, San-Yih Lin, and Chi-Wang Shu. TVB Runge-Kutta local projection discontinuous Galerkin finite element method for conservation laws III: One-dimensional systems. Journal of Computational Physics, 84(1):90-113, September 1989

[CMS10] Nicolas Crouseilles, Michel Mehrenberger, and Eric Sonnendrücker. Conservative semi-Lagrangian schemes for Vlasov equations. Journal of Computational Physics, 229(6):1927-1953, March 2010.

[CO09] Marco Caliari and Alexander Ostermann. Implementation of exponential Rosenbrocktype integrators. Applied Numerical Mathematics, 59(3-4):568-581, March 2009.

[CS89] Bernardo Cockburn and Chi-Wang Shu. TVB Runge-Kutta local projection discontinuous Galerkin finite element method for conservation laws II: General framework. Mathematics of Computation, 52:411-435, 1989.

[CS91] Bernardo Cockburn and Chi-Wang Shu. The Runge-Kutta local projection P1- 
[CS98a]

[CS98b]

[CVB04]

[FSB01]

[GBNS14]

[GQ13]

[GR08]

[GvL12]

HGMM12]

[HLS98]

[JP86]

[Kop09]

[Kra13]

[Law67]

[LC05]

[Le 13]

[MBGW13]

$\left[\mathrm{MSM}^{+} 13\right]$

[MvL06]

[NY99]

[QS11]

discontinuous-Galerkin finite element method for scalar conservation laws. RairoMathematical Modelling and Numerical Analysis, 25(3):337-361, 1991.

Bernardo Cockburn and Chi-Wang Shu. The local discontinuous Galerkin method for time-dependent convection-diffusion systems. Siam Journal On Numerical Analysis, 35(6):2440-2463, November 1998.

Bernardo Cockburn and Chi-Wang Shu. The Runge-Kutta discontinuous galerkin method for conservation laws V: Multidimensional systems. Journal of Computational Physics, 141(2):199-224, April 1998.

Marco Caliari, Marco Vianello, and Luca Bergamaschi. Interpolating discrete advection-diffusion propagators at Leja sequences. Journal of Computational and Applied Mathematics, 172(1):79-99, November 2004.

Tilman Dannert and Frank Jenko. Vlasov simulation of kinetic shear Alfvén waves. Computer Physics Communications, 163(2):67-78, 2004.

Francis Filbet, Eric Sonnendrücker, and Pierre Bertrand. Conservative numerical schemes for the Vlasov equation. Journal of Computational Physics, 172(1):166187, September 2001.

Ferran Garcia, Luca Bonaventura, Marta Net, and Juan Sánchez. Exponential versus IMEX high-order time integrators for thermal convection in rotating spherical shells. Journal of Computational Physics, 264(0):41-54, May 2014.

Wei Guo and Jing-Mei Qiu. Hybrid semi-Lagrangian finite element-finite difference methods for the Vlasov equation. Journal of Computational Physics, 234(0):108132, February 2013.

Francis X. Giraldo and Marco Restelli. A study of spectral element and discontinuous Galerkin methods for the Navier-Stokes equations in nonhydrostatic mesoscale atmospheric modeling: Equation sets and test cases. Journal of Computational Physics, 227(8):3849-3877, April 2008.

Gene H. Golub and Charles F. van Loan. Matrix Computations. JHU Press, 2012.

R. E. Heath, Irene M. Gamba, Philip J. Morrison, and C. Michler. A discontinuous Galerkin method for the Vlasov-Poisson system. Journal of Computational Physics, 231(4):1140-1174, February 2012.

Marlis Hochbruck, Christian Lubich, and Hubert Selhofer. Exponential integrators for large systems of differential equations. SIAM J. Sci. Comput., 19(5):1552-1574, 1998.

Claes Johnson and Juhani Pitkäranta. An analysis of the discontinuous Galerkin method for a scalar hyperbolic equation. Mathematics of Computation, 46(173):126, January 1986.

David A. Kopriva. Implementing Spectral Methods for Partial Differential Equations. Springer, 2009.

Michael Kraus. Variational integrators in plasma physics. Technical report, MaxPlanck-Institut für Plasmaphysik, 2013.

J. Douglas Lawson. Generalized Runge-Kutta processes for stable systems with large Lipschitz constants. SIAM J. Numer. Anal., 4(3):72-380, 1967.

Daniel Y. Le Roux and G. F. Carey. Stability/dispersion analysis of the discontinuous Galerkin linearized shallow-water system. Int. J. Numer. Meth. Fluids, 48(3):325347, 2005.

Daniel Y. Le Roux. Fourier analyses of high order continuous and discontinuous Galerkin methods. Imperial College, 2013.

Andreas Müller, Jörn Behrens, Francis X. Giraldo, and Volkmar Wirth. Comparison between adaptive and uniform discontinuous Galerkin simulations in dry 2D bubble experiments. Journal of Computational Physics, 235(0):371-393, February 2013.

Michel Mehrenberger, Christophe Steiner, Luca Marradi, Nicolas Crouseilles, Eric Sonnendrücker, and Bedros Afeyan. Vlasov on GPU (VOG project). ESAIM: PROCEEDINGS, 43:37-58, December 2013.

Cleve Moler and Charles van Loan. Nineteen dubious ways to compute the exponential of a matrix, twenty-five years later. SIAM Review, 45(1):3-49, 2006.

Takashi Nakamura and Takashi Yabe. Cubic interpolated propagation scheme for solving the hyper-dimensional Vlasov-Poisson equation in phase space. Computer Physics Communications, 120(23):122-154, August 1999.

Jing-Mei Qiu and Chi-Wang Shu. Positivity preserving semi-Lagrangian discontinuous Galerkin formulation: Theoretical analysis and application to the vlasov-poisson system. Journal of Computational Physics, 230(23):8386-8409, September 2011. 
[RBS06] Marco Restelli, Luca Bonaventura, and Riccardo Sacco. A semi-Lagrangian discontinuous Galerkin method for scalar advection by incompressible flows. Journal of Computational Physics, 216(1):195-215, July 2006.

[RG09] Marco Restelli and Francis X. Giraldo. A conservative discontinuous Galerkin semiimplicit formulation for the Navier-Stokes equations in nonhydrostatic mesoscale modeling. SIAM J. Sci. Comput., 31(3):2231-2257, January 2009.

[RH73] Wm. H. Reed and T. R. Hill. Triangular mesh methods for the neutron transport equation. Technical Report LA-UR-73-479, Los Alamos Scientific Laboratory Report, October 1973.

[RS11] Thomas Respaud and Eric Sonnendrücker. Analysis of a new class of forward semiLagrangian schemes for the 1D Vlasov-Poisson equations. Numerische Mathematik, 118(2):329-366-, 2011.

[RT77] Pierre-Arnaud Raviart and J. M. Thomas. A mixed finite element method fpr 2-nd order elliptic problems. Number 666 in Lecture Notes in Mathematics Series. Mathematical Aspects of Finite Element Methods, 1977.

[Saa91] Yousef Saad. Analysis of some Krylov subspace approximations to the matrix exponential operator. SIAM J. Numer. Anal., 29(1):209-228, 1991.

[Sho79] Magdi M. Shoucri. Nonlinear evolution of the bump-on-tail instability. Physics of Fluids (1958-1988), 22(10):2038-2039, 1979.

[SRBG99] Eric Sonnendrücker, Jean Roche, Pierre Bertrand, and Alain Ghizzo. The semiLagrangian method for the numerical resolution of the Vlasov equation. Journal of Computational Physics, 149(2):201-220, 1999.

[SSS09] Jan C. Schulze, Peter J. Schmid, and Jörn L. Sesterhenn. Exponential time integration using Krylov subspaces. Int. J. Numer. Meth. Fluids, 60(6):591-609, 2009.

[ZS10] Xiangxiong Zhang and Chi-Wang Shu. On maximum-principle-satisfying high order schemes for scalar conservation laws. Journal of Computational Physics, 229(9):3091-3120, May 2010. 